CERN-TH/2001-174

UPRF-2001-11

hep-ph/0107138

\title{
Soft-Gluon Resummation for the Fragmentation of Light and Heavy Quarks at Large $x$ "
}

\author{
Matteo Cacciari ${ }^{(1)}$ and Stefano Catani ${ }^{(2)}$ \\ (1) Dipartimento di Fisica, Università di Parma, Italy, and \\ INFN, Sezione di Milano, Gruppo Collegato di Parma \\ (2) CERN - Theory Division \\ CH 1211 Geneva 23, Switzerland
}

\begin{abstract}
We present a QCD study of fragmentation processes for light and heavy quarks in the semi-inclusive region of large $x$. Large logarithmic terms, due to soft-gluon radiation, are evaluated and resummed to all perturbative orders in the QCD coupling $\alpha_{\mathrm{S}}$. Complete analytical results to next-toleading logarithmic accuracy are given for one-particle and two-particle inclusive distributions in $e^{+} e^{-}$annihilation and DIS. Factorization of parton radiation at low transverse momenta is exploited to identify the universal (processindependent) perturbative fragmentation function that controls heavy-quark processes, and to perform next-to-leading logarithmic resummation of its softgluon contributions. To gauge the quantitative impact of resummation, we perform numerical studies of light- and heavy-quark fragmentation in $e^{+} e^{-}$ collisions.
\end{abstract}

CERN-TH/2001-174

July 2001

\footnotetext{
*This work was supported in part by the EU Fourth Framework Programme "Training and Mobility of Researchers", Network "Quantum Chromodynamics and the Deep Structure of Elementary Particles", contract FMRX-CT98-0194 (DG 12 - MIHT).

${ }^{\dagger}$ On leave of absence from INFN, Sezione di Firenze, Florence, Italy.
} 


\section{Introduction}

Large logarithmically-enhanced corrections due to soft-gluon radiation are a general feature in the perturbative-QCD study of hard-scattering processes near threshold. Techniques for resumming these corrections have been developed over the past several years and have been mainly applied to the production cross sections of high-mass systems. An extensive and updated list of references can be found in Sect. 5 of Ref. [1].

In spite of the large amount of available data from $e^{+} e^{-}$, lepton-hadron and hadronhadron collisions (see [2, 3] and references therein), soft-gluon effects in single-particle (and double-particle) inclusive cross sections have instead received less attention. In this paper we consider soft-gluon resummation for the fragmentation processes of light- and heavyflavoured hadrons in the vicinity of the threshold region.

The basis for higher-order calculations in perturbative QCD is provided by the factorization theorem of mass singularities [4]. According to it, any inclusive hard cross section $\sigma\left(x, Q^{2}\right)$, involving initial-state hadrons and detected final-state hadrons, can be written as follows

$$
\sigma\left(x, Q^{2}\right)=F \otimes \hat{\sigma} \otimes D+\mathcal{O}\left((\Lambda / Q)^{p}\right)
$$

The notation in Eq. (任) is symbolic: $Q^{2}$ is the hard scale, i.e. a typical transferred momentum much larger than the QCD scale $\Lambda^{2} ; x$ stands for any ratio of other kinematic invariants; the symbol $\otimes$ denotes appropriate convolutions of longitudinal- and transversemomentum variables and the sum over parton indices is understood. The term $\mathcal{O}\left((\Lambda / Q)^{p}\right)$ on the right-hand side of Eq. (11) stands for cross section contributions that are suppressed by some inverse power $p(p \geq 1)$ of $Q$ in the hard-scattering regime $Q \gg \Lambda$.

Perturbation theory allows us to evaluate the first term on the right-hand side of Eq. (11), that is, the so-called leading-twist component of the hard-scattering process. Performing a power series expansion in the strong coupling $\alpha_{\mathrm{S}}\left(Q^{2}\right)$, we can compute the partonic cross section $\hat{\sigma}$ and the $Q^{2}$-evolution of the parton distribution functions $F\left(x, Q^{2}\right)$ and of the parton fragmentation functions $D\left(x, Q^{2}\right)$. Note, in particular, that only the $Q^{2}$-dependence (or, more precisely, the anomalous dimensions) of the parton distributions $F\left(x, Q^{2}\right)$ and $D\left(x, Q^{2}\right)$ is under control within perturbation theory. Their absolute normalization at a given (and arbitrary) scale has to be provided as phenomenological input. In the perturbative calculation this arbitrariness is reflected by the fact that anomalous dimensions and coefficient functions are separately dependent on the factorization scheme. Any definite prediction thus requires a consistent evaluation of anomalous dimensions and coefficient functions within the same factorization scheme.

As long as all the kinematic scales are of the same order (i.e. $x=\mathcal{O}(1)$ ), perturbative calculations to the first few orders in the QCD coupling $\alpha_{\mathrm{S}}$ should provide reliable and accurate theoretical predictions for the hadronic cross section. However, the perturbative series for the anomalous dimensions and for the coefficient functions are poorly convergent in the semi-inclusive or Sudakov region, that is, when the energy or transverse momentum of the triggered hadron (final state) is a large fraction, $x \rightarrow 1$, of the available energy $\sqrt{s}$ in the scattering process. In this case the production threshold is approached and the emission of accompanying radiation is strongly inhibited by the kinematics. Only soft particles can 
be radiated in the inclusive final state, and the bremsstrahlung spectrum of soft (and collinear) gluons produces large logarithmic contributions of the type $\alpha_{\mathrm{S}}^{n} \ln ^{m}(1-x) /(1-x)$ (with $m \leq 2 n-1$ ) to each order $n$ in perturbation theory. In the presence of these contributions, the 'true' expansion parameter is no longer $\alpha_{\mathrm{S}}$ but rather the large effective coupling $\alpha_{\mathrm{S}} \ln ^{2}(1-x)$ and, hence, any finite-order perturbative calculation is unable to provide an accurate evaluation of the cross section. The only reliable procedure consists in resumming classes of logarithms to all orders in $\alpha_{\mathrm{S}}$.

Leading and next-to-leading logarithmic contributions to parton distributions and parton fragmentation functions are known. To be definite, let us consider the $\overline{\mathrm{MS}}$ factorization scheme and introduce the $N$ moments $D_{a / h, N}\left(Q^{2}\right)$ of the fragmentation function of the parton $a$ into the light hadron $h$ :

$$
D_{a / h, N}^{(\overline{\mathrm{MS}})}\left(Q^{2}\right) \equiv \int_{0}^{1} d x x^{N-1} D_{a / h}^{(\overline{\mathrm{MS}})}\left(x, Q^{2}\right) .
$$

The $Q^{2}$-evolution of the parton fragmentation functions is given by the Altarelli-Parisi (AP) equations

$$
\frac{d D_{a / h, N}^{(\overline{\mathrm{MS}})}\left(Q^{2}\right)}{d \ln Q^{2}}=\sum_{b} \gamma_{a b, N}\left(\alpha_{\mathrm{S}}\left(Q^{2}\right)\right) D_{b / h, N}^{(\overline{\mathrm{MS}})}\left(Q^{2}\right),
$$

where the anomalous dimensions $\gamma_{a b, N}$ are the $N$ moments of the AP probabilities. An important feature of the $\overline{\mathrm{MS}}$ factorization scheme is that only the flavour-diagonal tributions $\gamma_{q q}$ and $\gamma_{g g}$ to the evolution are affected by enhanced logarithmic corrections at large $N$ (or, equivalently, at large $x$ ). In particular, the explicit expressions of the flavour-diagonal anomalous dimensions are [5]

$$
\begin{aligned}
& \gamma_{q q, N}\left(\alpha_{\mathrm{S}}\right) \simeq-C_{F} \frac{\alpha_{\mathrm{S}}}{\pi}\left(1+K \frac{\alpha_{\mathrm{S}}}{2 \pi}+\mathcal{O}\left(\alpha_{\mathrm{S}}^{2}\right)\right) \ln N+\mathcal{O}(1) \\
& \gamma_{g g, N}\left(\alpha_{\mathrm{S}}\right) \simeq-C_{A} \frac{\alpha_{\mathrm{S}}}{\pi}\left(1+K \frac{\alpha_{\mathrm{S}}}{2 \pi}+\mathcal{O}\left(\alpha_{\mathrm{S}}^{2}\right)\right) \ln N+\mathcal{O}(1)
\end{aligned}
$$

where the coefficient $K$ [6] is given by

$$
K=C_{A}\left(\frac{67}{18}-\frac{\pi^{2}}{6}\right)-\frac{5}{9} N_{f},
$$

and the term $\mathcal{O}(1)$ denotes any non-singular contribution at large $N$. The expressions (目) and (5) also show another important feature of the $\overline{\mathrm{MS}}$ factorization scheme. In this scheme the anomalous dimensions are not more singular than a single power of $\ln N$ when $N \rightarrow \infty$ [8].

A similar result is valid for the parton distributions $F^{(\overline{\mathrm{MS}})}\left(x, Q^{2}\right)$, and the corresponding anomalous dimensions, in the large- $N$ limit, are again given by Eqs. (4) and (5).

\footnotetext{
$\ddagger$ In $N$-moment space, the semi-inclusive region $x \rightarrow 1$ corresponds to the limit $N \rightarrow \infty$.

$\S^{\S}$ The non-diagonal terms are suppressed by a relative factor of $\mathcal{O}(1 / N)$.

IIn this paper $\alpha_{\mathrm{S}}\left(Q^{2}\right)$ denotes the QCD coupling in the $\overline{\mathrm{MS}}$ renormalization scheme. The value of the coefficient $K$ reported in Eq. (6) refers to this renormalization scheme. One can also introduce [f] an alternative renormalization scheme such as to absorb the coefficient $K$ in the redefinition of $\alpha_{\mathrm{S}}$ according to $\alpha_{\mathrm{S}} \rightarrow \alpha_{\mathrm{S}}\left(1+K \alpha_{\mathrm{S}} / 2 \pi\right)$.
} 
The knowledge of the large- $N$ behaviour of the anomalous dimensions is however not sufficient to evaluate the hadronic cross section in the large- $x$ region. The consistency of the resummation procedure with the factorization theorem of mass singularities demands also the calculation, to the same logarithmic accuracy, of the process-dependent partonic cross section. The latter can strongly affect the hadronic cross section because (unlike the anomalous dimensions) the perturbative series for its $N$ moments $\hat{\sigma}_{N}$ contains doublelogarithmic terms $\alpha_{\mathrm{S}} \ln ^{2} N$ in the general form

$$
\hat{\sigma}_{N} \sim \hat{\sigma}_{N}^{(L O)}\left\{1+\sum_{n=1}^{\infty} \alpha_{\mathrm{S}}^{n} \sum_{m=1}^{2 n} c_{n, m} \ln ^{m} N\right\}
$$

where $\hat{\sigma}_{N}^{(L O)}$ is the leading-order (LO) contribution. Moreover, we should keep in mind that the soft-gluon contributions to the partonic cross section can be sizeable long before the threshold region in the hadronic cross section is actually approached. This is because the evolution of the parton densities and fragmentation functions sizeably reduces the energy that is available in the partonic hard-scattering subprocess; thus, the partonic cross section $\hat{\sigma}$ in the factorization formula (i) is typically evaluated much closer to threshold than the hadronic cross section.

In recent years, the general methods developed in Refs. [8, 9, 11 14 have been applied to carry out soft-gluon resummation to next-to-leading logarithmic accuracy for several processes. Nonetheless, no explicit resummed calculation has been performed for fragmentation cross sections.

In this paper, we consider fragmentation cross sections in $e^{+} e^{-}$annihilation and deep inelastic lepton-nucleon scattering (DIS). We resum leading and next-to-leading soft-gluon contributions to one-particle and two-particle inclusive distributions. In the case of the oneparticle distribution in $e^{+} e^{-}$, we also discuss some of the next-to-next-to-leading terms.

We also consider the fragmentation of heavy quarks. In the limit when the heavy-quark mass $m$ is much smaller than the hard scale $Q$ of the scattering process, the factorization formula (1) can be generalized in a process-independent way to compute heavy-quark cross sections. The generalization is based on the perturbative fragmentation function formalism [15, 16], which uses the AP evolution equations (3) to resum the large single-logarithmic contributions $\left(\alpha_{\mathrm{S}} \ln Q^{2} / m^{2}\right)^{n}$ of collinear origin. We shall show how mass and Sudakov effects can systematically be included in the AP evolution at low transverse momentum, thus extending the formalism of Refs. [15, 16] to include soft-gluon resummation at large $x$. Our results demonstrate that in the limit $m / Q \ll 1$, the soft-gluon contributions are process-independent and can thus be resummed in the universal perturbative component of the heavy-quark fragmentation function. In particular, we generalize the results of the soft-gluon resummed calculations performed in Refs. [15, 17] by evaluating the heavy-quark fragmentation function to next-to-leading logarithmic accuracy in the large- $x$ region.

The outline of the paper is as follows. In Section 2 we consider light-quark fragmentation. In Sect. 2.1 we provide analytical results for the next-to-leading Sudakov resummation of the $e^{+} e^{-}$coefficient function in the $\overline{\mathrm{MS}}$ scheme. In the same section we also discuss some next-to-next-to-leading terms, and point out a universality pattern by comparing with the DIS structure function case. In Sect. 2.2 we then perform some numerical studies and assess the impact of the resummation on the value of the $e^{+} e^{-}$single-inclusive distribution 


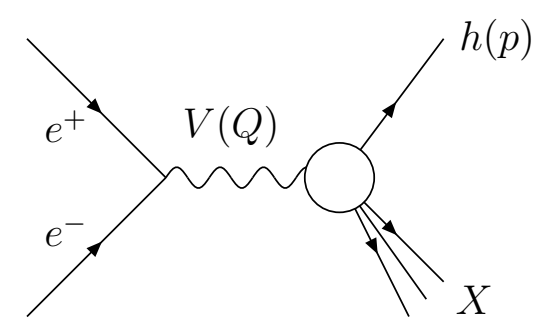

Figure 1: Inclusive production of a hadron $h$ with momentum $p$ in $e^{+} e^{-}$annihilation.

at large $x$, and on the stability of the result with respect to renormalization/factorization scale variations. In Section 3 we consider the case of heavy-quark fragmentation. We start in Sect. 3.1 by briefly reviewing the perturbative fragmentation function formalism. In Sect. 3.2 we show how the process-independent initial condition for the perturbative fragmentation function can be introduced by exploiting the universal factorization properties of parton radiation at low transverse momenta. Next-to-leading Sudakov resummation for the initial condition is performed in Sect. 3.3. The numerical studies performed in Sects. 3.3 and 3.4 assess the effects of Sudakov resummation on softening and scale dependence of the fragmentation function and single-inclusive $e^{+} e^{-}$distribution of heavy quarks. In Sect. 1 we finally summarize our main results. Soft-gluon resummation for two-particle distributions in $e^{+} e^{-}$collisions and one-hadron inclusive cross section in DIS is considered in Appendices $\mathrm{A}$ and $\mathrm{B}$, respectively.

\section{Light-quark fragmentation at large $x$}

In this section we consider the fragmentation of light quarks (light hadrons) by performing a detailed theoretical and numerical study of the single-particle inclusive cross section in $e^{+} e^{-}$ collisions. Related theoretical results on the two-particle distribution in $e^{+} e^{-}$annihilation and the single-inclusive cross section in DIS are presented in the appendices.

\subsection{Single-particle inclusive distribution in $e^{+} e^{-}$annihilation}

We consider the inclusive production of a single-hadron $h$ with momentum $p$ in $e^{+} e^{-}$ annihilation. Within the single-vector boson $\left(V=\gamma^{*}, Z^{0}\right)$ exchange approximation (Fig. 1),

$$
e^{+}+e^{-} \rightarrow V(Q) \rightarrow h(p)+X
$$

the single-particle angular distribution has three (transverse, asymmetric and longitudinal) components [18 20]. Each component can then be split in flavour singlet and flavour nonsinglet contributions. In the following we do not consider the longitudinal cross section or the 'pure' flavour-singlet contributions, because they are suppressed by a relative factor of 
the order of $(1-x)$ when $x \rightarrow 1$. The flavour non-singlet contributions can be written as

$$
\frac{d \sigma_{h}^{\left(e^{+} e^{-}\right)}\left(x, Q^{2}\right)}{d x}=\sigma^{(L O)} \int_{x}^{1} \frac{d z}{z} C^{\left(e^{+} e^{-}\right) \overline{\mathrm{MS}}}\left(x / z, \alpha_{\mathrm{S}}\left(\mu^{2}\right) ; Q^{2}, \mu^{2}, \mu_{F}^{2}\right) D^{(\overline{\mathrm{MS}})}\left(z, \mu_{F}^{2}\right)
$$

where $x$ is the fraction of the beam energy carried by the hadron,

$$
x=\frac{2 p \cdot Q}{Q^{2}}
$$

and $\mu$ and $\mu_{F}$ denote the renormalization and factorization scales, respectively. The expression (9) is valid for both the transverse and asymmetric cross sections: $\sigma^{(L O)}$ are the corresponding cross sections at LO and $D^{(\overline{\mathrm{MS}})}$ are the corresponding flavour non-singlet components of the quark fragmentation functions into the hadron $h$. In the following we consider the large- $x$ behaviour of the (flavour non-singlet) coefficient function $C^{\left(e^{+} e^{-}\right) \overline{\mathrm{MS}}}(x)$, and we do not make any distinction between transverse and asymmetric coefficient functions because they only differ by terms that are not singular in the limit $x \rightarrow 1$.

The perturbative calculation of the coefficient function" gives [18]:

$$
\begin{gathered}
C^{\left(e^{+} e^{-}\right)}\left(x, \alpha_{\mathrm{S}}\left(\mu^{2}\right) ; Q^{2}, \mu^{2}, \mu_{F}^{2}\right)=\delta(1-x)+\frac{\alpha_{\mathrm{S}}\left(\mu^{2}\right)}{\pi} C_{F}\left[\frac{1}{2}\left(\frac{1+x^{2}}{1-x}\right)+\ln \frac{Q^{2}}{\mu_{F}^{2}}\right. \\
\left.+\left(\frac{\ln (1-x)}{1-x}\right)_{+}-\frac{3}{4}\left(\frac{1}{1-x}\right)_{+}+\left(\frac{\pi^{2}}{3}-\frac{9}{4}\right) \delta(1-x)+f(x)\right]+\mathcal{O}\left(\alpha_{\mathrm{S}}^{2}\right)
\end{gathered}
$$

where $f(x)$ is a smooth function网 for $x \rightarrow 1$ and the $(\ldots)_{+}$-distributions are defined in the customary way:

$$
\int_{0}^{1} d z h(z)[g(z)]_{+} \equiv \int_{0}^{1} d z[h(z)-h(1)] g(z) .
$$

Introducing the $N$ moments, as in Eq. (2), the expression in Eq. (11) reads

$$
\begin{gathered}
C_{N}^{\left(e^{+} e^{-}\right)}\left(\alpha_{\mathrm{S}}\left(\mu^{2}\right) ; Q^{2}, \mu^{2}, \mu_{F}^{2}\right)=1+\frac{\alpha_{\mathrm{S}}\left(\mu^{2}\right)}{\pi} C_{F}\left[-\left(\ln N+\gamma_{E}-\frac{3}{4}\right) \ln \frac{Q^{2}}{\mu_{F}^{2}}+\frac{1}{2} \ln ^{2} N\right. \\
\left.+\left(\frac{3}{4}+\gamma_{E}\right) \ln N+\left(\frac{5}{12} \pi^{2}-\frac{9}{4}+\frac{1}{2} \gamma_{E}^{2}+\frac{3}{4} \gamma_{E}\right)+\mathcal{O}\left(\frac{1}{N}\right)\right]+\mathcal{O}\left(\alpha_{\mathrm{S}}^{2}\right)
\end{gathered}
$$

where $\gamma_{E}=0.5772 \ldots$ is the Euler constant. The large (when $N \rightarrow \infty$ ) contributions $\ln ^{k+1} N$ in Eq. (13) are due to the Mellin transformation of the singular (when $x \rightarrow 1$ ) distributions $\left[\ln ^{k}(1-x) /(1-x)\right]_{+}$, associated to the bremsstrahlung spectrum of soft and collinear emission. To higher orders in perturbation theory, the large- $N$ moments of the coefficient function have the general double-logarithmic expansion on the right-hand side of Eq. (7).

To perform the all-order resummation of the large $\ln N$ contributions, we can apply the general method developed in Ref. [9]. The single-particle distribution in Eq. (9) is obtained

\footnotetext{
${ }^{\|}$From now on, we omit the label $\overline{\mathrm{MS}}$ and we always use the $\overline{\mathrm{MS}}$ factorization scheme, unless explicitly stated otherwise.

** To be precise, $f(x) \sim \ln (1-x)$ when $x \rightarrow 1$, and thus the contribution of $f(x)$ to the right-hand side of Eq. (11) is less singular than that of the other distributions in the square bracket.
} 
by crossing to the final state the momentum of the incoming quark in the DIS process. Thus, we repeat step by step and in a straightforward manner the calculation of the DIS cross section carried out in Ref. [9]; we obtain the following resummed expression for the $e^{+} e^{-}$coefficient function:

$$
\begin{aligned}
\ln C_{N}^{\left(e^{+} e^{-}\right)}\left(\alpha_{\mathrm{S}}\left(\mu^{2}\right) ; Q^{2}, \mu^{2}, \mu_{F}^{2}\right) & =\ln \Delta_{N}\left(\alpha_{\mathrm{S}}\left(\mu^{2}\right), Q^{2} / \mu^{2} ; Q^{2} / \mu_{F}^{2}\right) \\
& +\ln J_{N}\left(\alpha_{\mathrm{S}}\left(\mu^{2}\right), Q^{2} / \mu^{2}\right)+\mathcal{O}\left(\alpha_{\mathrm{S}}\left(\alpha_{\mathrm{S}} \ln N\right)^{k}\right) .
\end{aligned}
$$

The radiative factors $\Delta_{N}$ and $J_{N}$ have the following exponentiated form:

$$
\begin{aligned}
\Delta_{N}\left(\alpha_{\mathrm{S}}\left(\mu^{2}\right), Q^{2} / \mu^{2} ; Q^{2} / \mu_{F}^{2}\right)= & \exp \left\{\int_{0}^{1} d z \frac{z^{N-1}-1}{1-z} \int_{\mu_{F}^{2}}^{(1-z)^{2} Q^{2}} \frac{d q^{2}}{q^{2}} A\left[\alpha_{\mathrm{S}}\left(q^{2}\right)\right]\right\}, \\
J_{N}\left(\alpha_{\mathrm{S}}\left(\mu^{2}\right), Q^{2} / \mu^{2}\right)= & \exp \left\{\int _ { 0 } ^ { 1 } d z \frac { z ^ { N - 1 } - 1 } { 1 - z } \left[\int_{(1-z)^{2} Q^{2}}^{(1-z) Q^{2}} \frac{d q^{2}}{q^{2}} A\left[\alpha_{\mathrm{S}}\left(q^{2}\right)\right]\right.\right. \\
& \left.\left.+\frac{1}{2} B\left[\alpha_{\mathrm{S}}\left((1-z) Q^{2}\right)\right]\right]\right\},
\end{aligned}
$$

where the functions $A\left(\alpha_{\mathrm{S}}\right)$ and $B\left(\alpha_{\mathrm{S}}\right)$ have perturbative expansions in $\alpha_{\mathrm{S}}$,

$$
\begin{aligned}
& A\left(\alpha_{\mathrm{S}}\right)=\sum_{n=1}^{\infty}\left(\frac{\alpha_{\mathrm{S}}}{\pi}\right)^{n} A^{(n)}, \\
& B\left(\alpha_{\mathrm{S}}\right)=\sum_{n=1}^{\infty}\left(\frac{\alpha_{\mathrm{S}}}{\pi}\right)^{n} B^{(n)},
\end{aligned}
$$

whose first coefficients are:

$$
\begin{aligned}
& A^{(1)}=C_{F}, \quad A^{(2)}=\frac{1}{2} C_{F} K=\frac{1}{2} C_{F}\left[C_{A}\left(\frac{67}{18}-\frac{\pi^{2}}{6}\right)-\frac{5}{9} N_{f}\right], \\
& B^{(1)}=-\frac{3}{2} C_{F} .
\end{aligned}
$$

Performing the integrations over $z$ and $q^{2}$ in Eqs. (15) and (16), we obtain a series of logarithmic contributions of the type $\alpha_{\mathrm{S}}^{n} \ln ^{m} N$, with $m \leq(n+1)$. We define as leading logarithmic (LL) the terms with $m=n+1$. The next-to-leading logarithmic (NLL) contributions are those with $m=n$, the next-to-next-to-leading logarithmic (NNLL) terms have $m=n-1$, and so forth.

Owing to the knowledge of the LL coefficient $A^{(1)}$ and of the NLL coefficients $A^{(2)}, B^{(1)}$ in Eqs. (19) and (20), the result in Eq. (14) resums all the leading and next-to-leading $\ln N$ contributions to the $N$ moments of the coefficient function of the single-particle distribution in $e^{+} e^{-}$annihilation.

Note that, according to Eqs. (14) and (15), in the large- $N$ limit, the $e^{+} e^{-}$coefficient function has the following dependence on the factorization scale $\mu_{F}$ :

$$
\frac{d \ln C_{N}^{\left(e^{+} e^{-}\right)}\left(\alpha_{\mathrm{S}}\left(\mu^{2}\right) ; Q^{2}, \mu^{2}, \mu_{F}^{2}\right)}{d \ln \mu_{F}^{2}} \simeq-A\left[\alpha_{\mathrm{S}}\left(\mu_{F}^{2}\right)\right] \int_{0}^{1} d z \frac{z^{N-1}-1}{1-z}
$$


Since, to the logarithmic accuracy of Eqs. (14) and (4), the (non-singlet) quark anomalous dimension $\gamma_{q q, N}\left(\alpha_{\mathrm{S}}\right)$ can be written as

$$
\gamma_{N}\left(\alpha_{\mathrm{S}}\right) \simeq A\left(\alpha_{\mathrm{S}}\right) \int_{0}^{1} d z \frac{z^{N-1}-1}{1-z} \simeq-A\left(\alpha_{\mathrm{S}}\right)[\ln N+\mathcal{O}(1)]
$$

Eq. (21) shows that the $\mu_{F}$-dependence of the coefficient function $C_{N}^{\left(e^{+} e^{-}\right)}$consistently matches (and thus cancels) the $\mu_{F}$-dependence of the fragmentation function $D_{N}^{(\overline{\mathrm{MS}})}$ in Eq. (9).

The NLL resummed result in Eq. (14) has a simple physical interpretation [7, 9] in terms of independent fragmentation of the observed hadron $h$ and of the recoiling jet. The radiative factor $\Delta_{N}$ describes the energy loss of the primary quark (or antiquark) that eventually fragments into the triggered hadron. This factor takes into account final-state radiation of gluons that are soft (i.e. with energy fraction $\omega / p_{0} \equiv 1-z \leq 1-x \sim 1 / N \ll 1$ ) and collinear (i.e. with small transverse momentum $q \sim \omega \theta=(1-z) p_{0} \theta \ll(1-z) Q$ ) with respect to the momentum $p^{\mu}$ of the observed hadron. Having fixed the energy of the jet that produces the observed hadron, the recoiling jet is constrained to carry a small invariant mass squared $k^{2}=(1-z) Q^{2} \lesssim(1-x) Q^{2} \ll 1$. The radiative factor $J_{N}$ describes the fragmentation of the invariant mass of the recoiling jet as produced by the final-state radiation of collinear (either soft or hard) partons.

This independent-fragmentation picture is an effective physical picture. Although the two jets do not evolve independently (classically), the quantum interferences due to noncollinear parton radiation cancel up to NLL accuracy. The destructive interference of soft-parton radiation at large angles follows from QCD coherence [21], but it is no longer complete beyond NLL order. The resummed expression in Eq. (14) can be extended to any logarithmic order as 11 14

$$
\begin{aligned}
C_{N}^{\left(e^{+} e^{-}\right)}\left(\alpha_{\mathrm{S}}\left(\mu^{2}\right) ; Q^{2}, \mu^{2}, \mu_{F}^{2}\right) & =c\left(\alpha_{\mathrm{S}}\left(\mu^{2}\right), Q^{2} / \mu^{2} ; Q^{2} / \mu_{F}^{2}\right) \Delta_{N}^{(\mathrm{int})}\left(\alpha_{\mathrm{S}}\left(\mu^{2}\right), Q^{2} / \mu^{2}\right) \quad \text { (23) } \\
& \Delta_{N}\left(\alpha_{\mathrm{S}}\left(\mu^{2}\right), Q^{2} / \mu^{2} ; Q^{2} / \mu_{F}^{2}\right) J_{N}\left(\alpha_{\mathrm{S}}\left(\mu^{2}\right), Q^{2} / \mu^{2}\right)+\mathcal{O}(1 / N) .
\end{aligned}
$$

This expression contains two other factors in addition to those of Eq. (14). The factor $c\left(\alpha_{\mathrm{S}}\right)$ does not depend on $N$, and it is due to radiative corrections produced by hard (with energy $E \sim Q$ ) virtual partons. This factor is computable as a power series expansion in $\alpha_{\mathrm{S}}$

$$
c\left(\alpha_{\mathrm{S}}\left(\mu^{2}\right), Q^{2} / \mu^{2} ; Q^{2} / \mu_{F}^{2}\right)=1+\sum_{n=1}^{\infty}\left(\frac{\alpha_{\mathrm{S}}}{\pi}\right)^{n} c^{(n)}\left(Q^{2} / \mu^{2} ; Q^{2} / \mu_{F}^{2}\right) .
$$

The radiative factor $\Delta_{N}^{(\mathrm{int})}$ is given by

$$
\Delta_{N}^{(\text {int })}\left(\alpha_{\mathrm{S}}\left(\mu^{2}\right), Q^{2} / \mu^{2}\right)=\exp \left\{\int_{0}^{1} d z \frac{z^{N-1}-1}{1-z} D\left[\alpha_{\mathrm{S}}\left((1-z)^{2} Q^{2}\right)\right]\right\}
$$

where

$$
D\left(\alpha_{\mathrm{S}}\right)=\left(\frac{\alpha_{\mathrm{S}}}{\pi}\right)^{2} D^{(2)}+\sum_{n=3}^{\infty}\left(\frac{\alpha_{\mathrm{S}}}{\pi}\right)^{n} D^{(n)} .
$$

Note that $\Delta_{N}^{\text {(int) }}$ embodies $\ln N$ contributions, and that the perturbative function $D\left(\alpha_{\mathrm{S}}\right)$ in Eq. (26) has a vanishing first-order coefficient $D^{(1)}$. Thus, $\Delta_{N}^{(\text {int })}$ contributes to the $e^{+} e^{-}$ 
coefficient function $C_{N}^{\left(e^{+} e^{-}\right)}$starting from NNLL order. This radiative factor takes into account soft-parton radiation at large angle (or, equivalently, with transverse momenta $q \sim \omega \theta \sim(1-z) Q$ ) and it leads to violation (at NNLL accuracy) of the "independentfragmentation' picture discussed above.

It is straightforward to check that our NLL result in Eq. (14) agrees with the large- $N$ limit of the exact $\mathcal{O}\left(\alpha_{\mathrm{S}}^{2}\right)$ calculation of Ref. [20]. Moreover, this calculation can also be used to extract a linear combination of the NNLL coefficients $B^{(2)}$ and $D^{(2)}$ in Eqs. (18) and (26). We find

$$
\begin{aligned}
D^{(2)}+\frac{1}{2} B^{(2)} & =\frac{1}{16}\left[C_{F}^{2}\left(-\frac{3}{2}+2 \pi^{2}-24 \zeta_{3}\right)+C_{F} C_{A}\left(-\frac{3155}{54}+\frac{22}{9} \pi^{2}+40 \zeta_{3}\right)\right. \\
& \left.+C_{F} N_{f}\left(\frac{247}{27}-\frac{4}{9} \pi^{2}\right)\right],
\end{aligned}
$$

where $\zeta_{n}$ is the Riemann zeta function $\left(\zeta_{3}=1.202 \ldots\right)$. An independent calculation of $B^{(2)}$ and $D^{(2)}$ could be performed by exploiting infrared-factorization formulae [22, 23] at $\mathcal{O}\left(\alpha_{\mathrm{S}}^{2}\right)$ (see, e.g., the analogous calculations carried out in Refs. [24, 25]). The knowledge of the remaining NNLL coefficient $A^{(3)}$ in Eq. (17) requires the $\mathcal{O}\left(\alpha_{\mathrm{S}}^{3}\right)$-calculation of the anomalous dimensions $\gamma_{q q, N}\left(\alpha_{\mathrm{S}}\right)$ that control the evolution of the $\overline{\mathrm{MS}}$ fragmentation functions.

It is interesting to compare the results in Eqs. (14) and (23) with the corresponding resummed expression, given in Refs. [7, 9], of the coefficient function for the DIS structure functions at large values of the Bjorken variable $x$. The comparison shows that, not only the anomalous dimensions of the parton distributions and fragmentation functions but also the coefficient functions exactly coincide in the semi-inclusive limit to NLL accuracy. Moreover, the lowest-order NNLL coefficient in Eq. (27) also coincides with the corresponding coefficient for the DIS process [26]. This correspondence extends, beyond the leading collinear level, the validity of the Gribov-Lipatov perturbative relation [27] between DIS structure functions and $e^{+} e^{-}$fragmentation functions.

\subsection{Numerical results}

We present some numerical results to illustrate the main quantitative effects of soft-gluon resummation on the single-particle distribution in $e^{+} e^{-}$annihilation. Since the $\mathcal{O}\left(\alpha_{\mathrm{S}}^{3}\right)$ anomalous dimensions (and the NNLL coefficient $A^{(3)}$ ) are not known, we limit ourselves to considering NLL resummation at large- $x$ matched to the complete next-to-leading order (NLO) calculation [18, 19] of the coefficient function.

Introducing the first two coefficients, $b_{0}$ and $b_{1}$, of the QCD $\beta$-function,

$$
b_{0}=\frac{11 C_{A}-4 T_{R} N_{f}}{12 \pi}, \quad b_{1}=\frac{17 C_{A}^{2}-10 C_{A} T_{R} N_{f}-6 C_{F} T_{R} N_{f}}{24 \pi^{2}}
$$

${ }^{\dagger}$ Note that the normalization of our coefficients $B^{(2)}$ and $D^{(2)}$ is different from that of the DIS coefficients in Ref. [26]. More precisely, the combination $\left(D^{(2)}+B^{(2)}\right)_{D I S}$ in Eq. (22) of Ref. [26] corresponds to our combination $16\left(D^{(2)}+B^{(2)} / 2\right)$. 
in terms of which we have

$$
\alpha_{\mathrm{S}}\left(\mu^{2}\right)=\frac{1}{b_{0} \ln \left(\mu^{2} / \Lambda^{2}\right)}\left(1-\frac{b_{1} \ln \ln \left(\mu^{2} / \Lambda^{2}\right)}{b_{0}^{2} \ln \left(\mu^{2} / \Lambda^{2}\right)}\right),
$$

and defining the variable $\lambda$,

$$
\lambda=b_{0} \alpha_{\mathrm{S}}\left(\mu^{2}\right) \ln N,
$$

we first evaluate the radiative factors in Eqs. (15) and (16) at NLL accuracy[ and we obtain:

$$
\begin{aligned}
\ln \Delta_{N}\left(\alpha_{\mathrm{S}}\left(\mu^{2}\right), Q^{2} / \mu^{2} ; Q^{2} / \mu_{F}^{2}\right) & =\ln N h^{(1)}(\lambda) \\
& +h^{(2)}\left(\lambda, Q^{2} / \mu^{2} ; Q^{2} / \mu_{F}^{2}\right)+\mathcal{O}\left(\alpha_{\mathrm{S}}\left(\alpha_{\mathrm{S}} \ln N\right)^{k}\right) \\
\ln J_{N}\left(\alpha_{\mathrm{S}}\left(\mu^{2}\right), Q^{2} / \mu^{2}\right) & =\ln N f^{(1)}(\lambda) \\
& +f^{(2)}\left(\lambda, Q^{2} / \mu^{2}\right)+\mathcal{O}\left(\alpha_{\mathrm{S}}\left(\alpha_{\mathrm{S}} \ln N\right)^{k}\right) .
\end{aligned}
$$

The LL and NLL functions $h^{(1)}, f^{(1)}$ and $h^{(2)}, f^{(2)}$ are given, in terms of the perturbative coefficients $A^{(1)}, A^{(2)}, B^{(1)}, b_{0}, b_{1}$, in Eqs. (75)-(78) of Ref. [14].

The Sudakov-resummed part $C_{N}^{S}$ of the $e^{+} e^{-}$coefficient function is then written (from Eq. (23)) at NLL accuracy as

$$
\begin{gathered}
C_{N}^{S}\left(\alpha_{\mathrm{S}}\left(\mu^{2}\right) ; Q^{2}, \mu^{2}, \mu_{F}^{2}\right)=\left\{1+\frac{\alpha_{\mathrm{S}}\left(\mu^{2}\right)}{\pi} C_{F}\left[\frac{5}{12} \pi^{2}-\frac{9}{4}+\frac{1}{2} \gamma_{E}^{2}+\frac{3}{4} \gamma_{E}\right.\right. \\
\left.\left.+\left(\frac{3}{4}-\gamma_{E}\right) \ln \frac{Q^{2}}{\mu_{F}^{2}}\right]\right\} \cdot \exp \left[\ln N g^{(1)}(\lambda)+g^{(2)}\left(\lambda, Q^{2} / \mu^{2} ; Q^{2} / \mu_{F}^{2}\right)\right],
\end{gathered}
$$

where

$$
\begin{aligned}
g^{(1)}(\lambda) & =h^{(1)}(\lambda)+f^{(1)}(\lambda)=\frac{A^{(1)}}{\pi b_{0} \lambda}[\lambda+(1-\lambda) \ln (1-\lambda)], \\
g^{(2)}\left(\lambda, Q^{2} / \mu^{2} ; Q^{2} / \mu_{F}^{2}\right) & =h^{(2)}\left(\lambda, Q^{2} / \mu^{2} ; Q^{2} / \mu_{F}^{2}\right)+f^{(2)}\left(\lambda, Q^{2} / \mu^{2}\right) \\
& =\frac{A^{(1)} b_{1}}{2 \pi b_{0}^{3}}\left[2 \lambda+2 \ln (1-\lambda)+\ln ^{2}(1-\lambda)\right]+\frac{\left(B^{(1)}-2 A^{(1)} \gamma_{E}\right)}{2 \pi b_{0}} \ln (1-\lambda) \\
& -\frac{1}{\pi b_{0}}[\lambda+\ln (1-\lambda)]\left(\frac{A^{(2)}}{\pi b_{0}}-A^{(1)} \ln \frac{Q^{2}}{\mu^{2}}\right)-\frac{A^{(1)}}{\pi b_{0}} \lambda \ln \frac{Q^{2}}{\mu_{F}^{2}} .
\end{aligned}
$$

and the term in the curly bracket is the constant (when $N \rightarrow \infty$ ) part of the coefficient function at $\mathcal{O}\left(\alpha_{\mathrm{S}}\right)$ (see Eq. $(\underline{13})$ ).

Our final expression for the $N$ moments of the coefficient function is

$$
\begin{aligned}
C_{N}^{(\mathrm{res})}\left(\alpha_{\mathrm{S}}\left(\mu^{2}\right) ; Q^{2}, \mu^{2}, \mu_{F}^{2}\right) & =C_{N}^{S}\left(\alpha_{\mathrm{S}}\left(\mu^{2}\right) ; Q^{2}, \mu^{2}, \mu_{F}^{2}\right)-\left[C_{N}^{S}\left(\alpha_{\mathrm{S}}\left(\mu^{2}\right) ; Q^{2}, \mu^{2}, \mu_{F}^{2}\right)\right]_{\alpha_{\mathrm{S}}} \\
& +\left[C_{N}^{\left(e^{+} e^{-}\right)}\left(\alpha_{\mathrm{S}}\left(\mu^{2}\right) ; Q^{2}, \mu^{2}, \mu_{F}^{2}\right)\right]_{\alpha_{\mathrm{S}}},
\end{aligned}
$$

where $\left[C_{N}^{\left(e^{+} e^{-}\right)}\right]_{\alpha_{\mathrm{S}}}$ is the full $e^{+} e^{-}$coefficient function at $\mathcal{O}\left(\alpha_{\mathrm{S}}\right)[18,19], C_{N}^{S}$ is given in Eq. (33) and $\left[C_{N}^{S}\right]_{\alpha_{S}}$ represents its perturbative truncation at $\mathcal{O}\left(\alpha_{\mathrm{S}}\right)$ (i.e. at NLO). Owing to the

*In our numerical calculations we always use the value $\Lambda^{(5)}=200 \mathrm{MeV}$ for the QCD scale $\Lambda^{(5)}$ with $N_{f}=5$ effective massless flavours. This corresponds to $\alpha_{\mathrm{S}}\left(M_{Z}^{2}\right)=0.116$.

${ }^{\dagger}$ This is achieved [9] by replacing $z^{N-1}-1 \rightarrow-\Theta\left(1-\frac{e^{-\gamma_{E}}}{N}-z\right)$ in the integrand. 


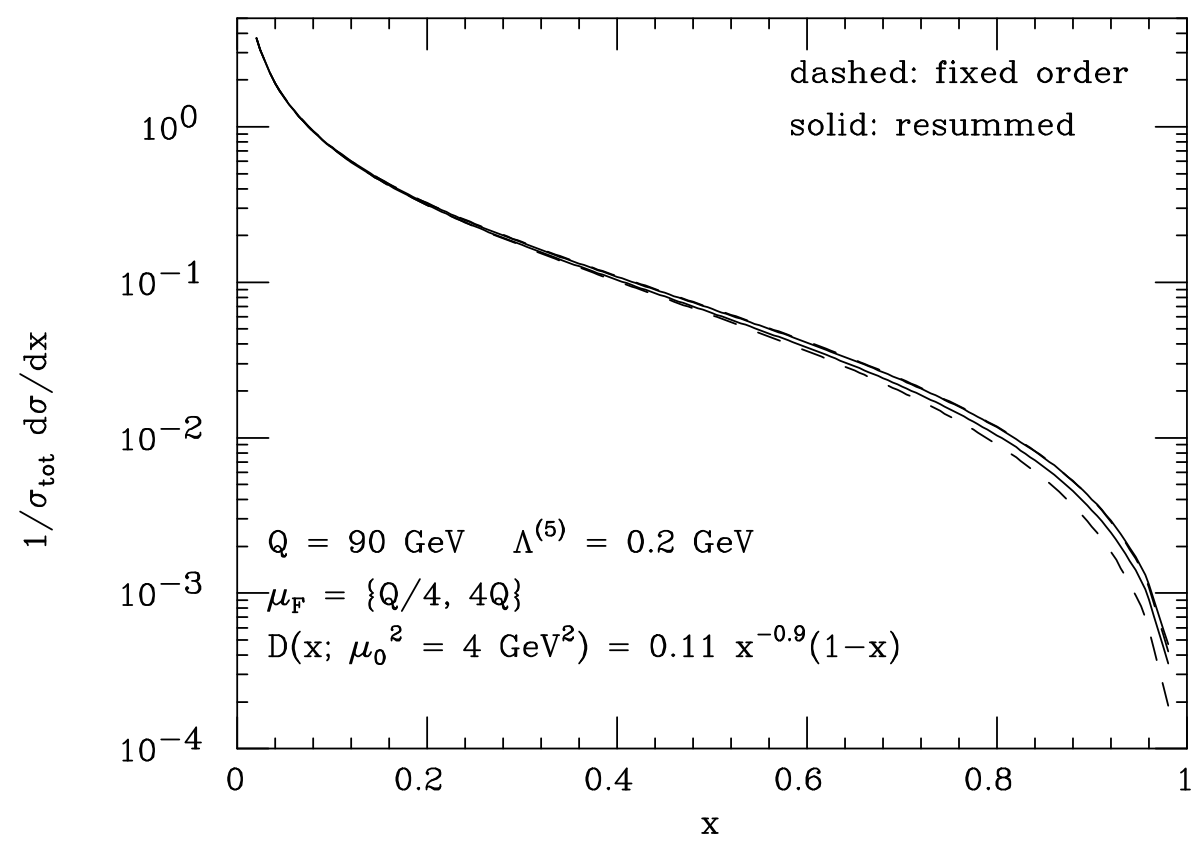

Figure 2: Effect of Sudakov resummation on a typical light-hadron fragmentation spectrum in $e^{+} e^{-}$collisions. The single-particle distribution is computed at NLO (dashed lines) and at NLO+NLL accuracy (solid lines). The factorization and renormalization scales are varied in the range $Q / 4 \leq \mu_{F}=\mu \leq 4 Q$.

subtraction applied to the resummed part $C_{N}^{S}$ on the right-hand side, Eq. (36) exactly reproduces the NLO result and resums soft-gluon effects beyond $\mathcal{O}\left(\alpha_{\mathrm{S}}\right)$ to NLL accuracy. This defines our NLO+NLL resummed calculation.

To obtain the single-particle inclusive cross section in Eq. (9), the $N$ moments of the coefficient function have to be multiplied by the $N$ moments $D_{N}\left(\mu_{F}^{2}\right)$ of the parton fragmentation functions, and then we have to perfom the inverse Mellin transformation to the $x$ space. Note that the resummed part of the $N$ moments of the coefficient function has cut singularities that start at the branch-point $N=N_{L}=\exp \left(1 / b_{0} \alpha_{\mathrm{S}}\right)$ (i.e. at $\lambda=1$ in Eqs. (34) and (35)) in the complex variable $N$. These singularities, which are related to the divergent behaviour of the running coupling $\alpha_{\mathrm{S}}\left(q^{2}\right)$ near the Landau pole at $q=\Lambda$, signal the onset of non-perturbative phenomena at very large values of $N$ or, equivalently, when $x$ is very close to its threshold value $x=1$. As discussed in detail in Ref. [28], if we are not interested in very high (small) values of $x\left(Q^{2}\right)$, we can avoid the explicit introduction of non-perturbative effects to deal with the Landau singularity. We thus use the Minimal Prescription of Ref. [28]: the inverse Mellin transformation is performed in the complex $N$ plane by choosing an integration contour that has all the singularities on its left, except for the singularity at $N=N_{L}$, which should lie far on its right. We numerically carry out the inverse Mellin transformation along this contour.

We present numerical results for the single inclusive (transverse plus longitudinal) distribution $1 / \sigma_{\text {tot }} d \sigma / d x$ in $e^{+} e^{-}$annihilation, where the total hadronic cross section $\sigma_{\text {tot }}$ is 


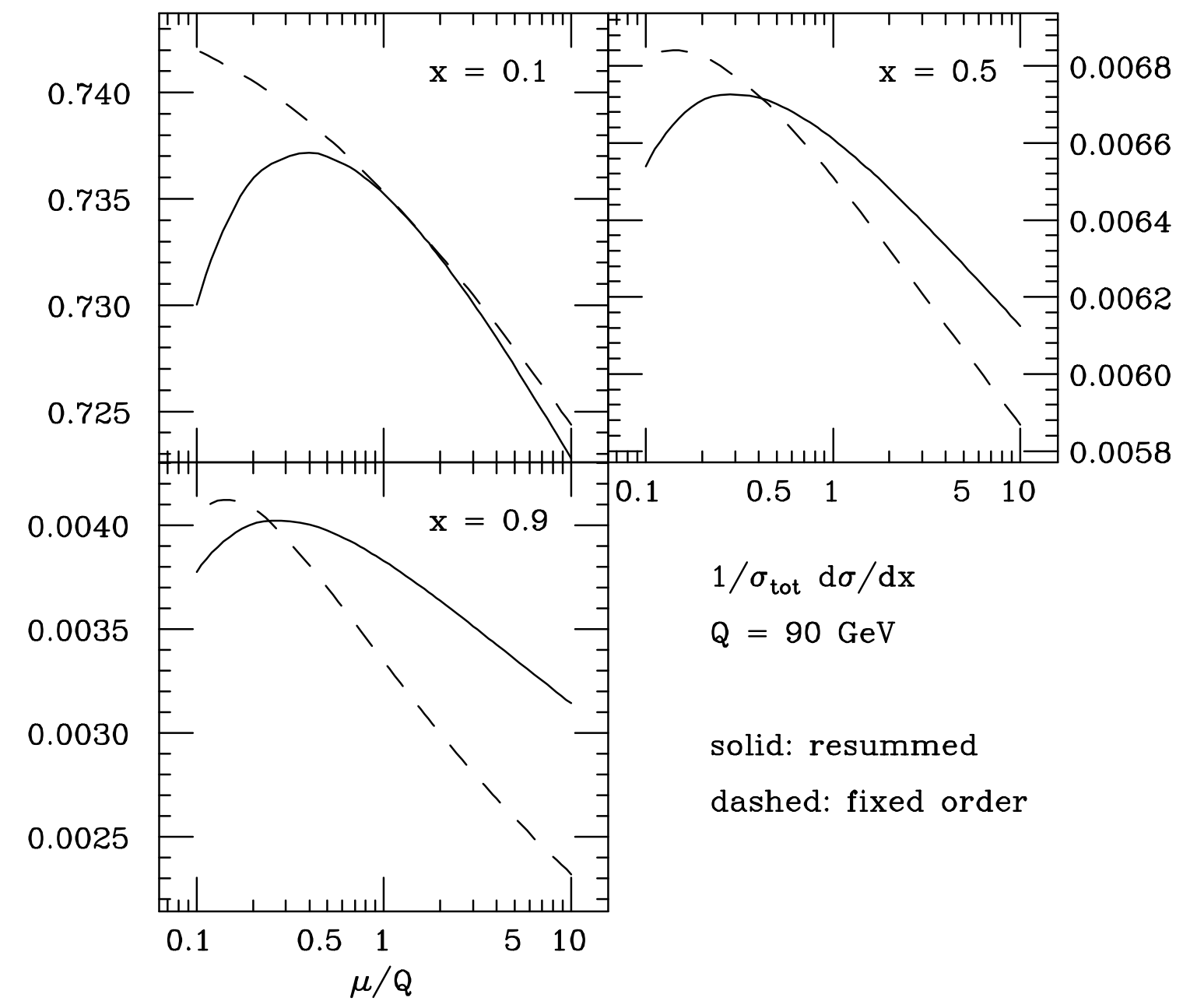

Figure 3: Dependence of the light-hadron fragmentation spectrum shown in Fig. 2 on the factorization/renormalization scales $\mu=\mu_{F}$, at three different valules of $x$.

evaluated at NLO:

$$
\sigma_{\text {tot }}\left(Q^{2}\right)=\sigma^{(L O)}\left(Q^{2}\right)\left[1+\frac{\alpha_{\mathrm{S}}\left(Q^{2}\right)}{\pi}+\mathcal{O}\left(\alpha_{\mathrm{S}}^{2}\right)\right] .
$$

For our illustrative purpose, we have considered only the non-singlet component of the distribution, and we have chosen a typical $x$-spectrum [29], $D\left(x ; \mu_{0}^{2}\right)=0.11 x^{-0.9}(1-x)$ (normalized by $\int_{0}^{1} d x D\left(x ; \mu_{0}^{2}\right)=1$ ), for the fragmentation function at the input scale $\mu_{0}=$ $2 \mathrm{GeV}$. The input fragmentation function is evolved up to the scale $\mu_{F}$ by using the NLO AP equation in the non-singlet channel (see Eq. (43) for details), and then it is convoluted with the $e^{+} e^{-}$coefficient function. The (non-singlet) coefficient function is evaluated either at NLO or at NLO+NLL accuracy. The renormalization $(\mu)$ and factorization $\left(\mu_{F}\right)$ scales are fixed to be equal and are varied within the range $Q / 4 \leq \mu \leq 4 Q$.

The NLO and resummed calculations at the centre-of-mass energy $Q=90 \mathrm{GeV}$ are compared in Fig. 2. As expected, the results of the two calculations only differ at large $x$. In the large- $x$ region the resummed calculation has a reduced dependence on the scale $\mu$. Moreover, at fixed scale $\mu=Q$ and fixed input of the fragmentation function, soft-gluon 


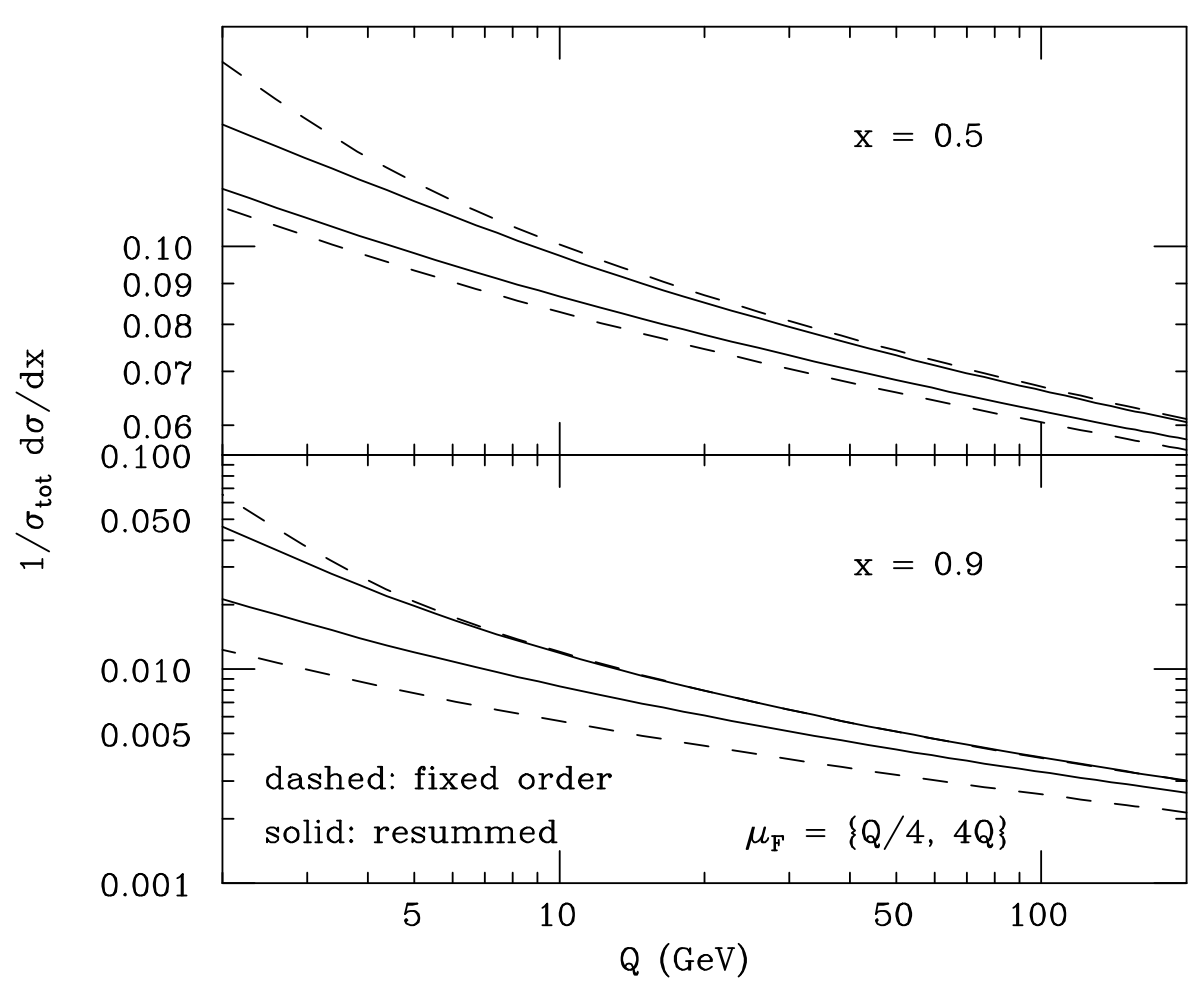

Figure 4: Scaling violations at $x=0.5$ and $x=0.9$, and uncertainty bands due to variations of the renormalization/factorization scales $\left(\mu=\mu_{F}\right)$. The fragmentation function at the input scale $\mu_{0}$ is the same as in Fig. 2 .

resummation increases the value of the single-particle distribution. This resummation effect can be mimicked by lowering the value of $\mu$ in the NLO calculation.

The enhancement of the distribution in the resummed calculation can appear surprising, since the suppression of radiation near threshold is physically expected to decrease the cross section. The apparent contradiction is due to the actual definition of the fragmentation function and coefficient function. The effect of inhibiting soft-gluon radiation is included in part in the AP evolution of the fragmentation function and in part in the coefficient function, and the separation depends on the factorization scheme. It turns out that, in the $\overline{\mathrm{MS}}$ factorization scheme, the AP evolution overestimates the physical effect of soft-gluon suppression. This overestimate is included in both the NLO and NLO+NLL calculations. The additional contributions that are resummed in the NLO+NLL case are those soft-gluon effects that are left in the coefficient function after factorization of the evolved fragmentation function. Having included too much soft-gluon suppression in the $\overline{\mathrm{MS}}$ evolution, the residual effect in the coefficient function is positive and tends to enhance the fixed-order perturbative distribution.

More numerical studies on the effect of resummation are shown in Figs. 3 and 4 .

The plots in Fig. 3 show the dependence of the fixed-order (dashed line) and resummed results (solid line) on the renormalization/factorization scales $\mu=\mu_{F}$, at the three points $x=0.1,0.5,0.9$. As expected from Fig. 2, the NLO+NLL calculation is more stable and higher (when $\mu \sim Q$ ) than the NLO one at large values of $x$. It is worth noting that the 
agreement of the two calculations at $x=0.1$ actually looks restricted to the region $\mu \simeq Q$. However this statement, and in general the $\mu$-dependence at $x \lesssim 0.1$, can strongly depend on the detailed shape of the input distribution $D\left(x ; \mu_{0}^{2}\right)$.

The effect of resummation as a function of the centre-of-mass energy $Q$ is shown in Fig. 4 , where uncertainty bands are presented for the scaling violations of the $e^{+} e^{-}$fragmentation spectrum at $x=0.5$ and $x=0.9$. Lowering the energy $Q$, the difference between the resummed and fixed-order calculations is enhanced by the increase of $\alpha_{\mathrm{S}}(Q)$. The bands of the resummed calculation are uniformly narrower, indicating that the NLO+NLL result is indeed more reliable than the NLO one.

These results on soft-gluon resummation for the $\overline{\mathrm{MS}}$ coefficient function in $e^{+} e^{-}$collisions can be implemented in global fits 29 of $e^{+} e^{-}$data to extract parton fragmentation functions. In particular, the reduced scale dependence of the NLO+NLL calculation can improve the determination of the quark fragmentation functions at large $x$.

\section{Heavy-quark fragmentation}

In this section we consider the fragmentation of heavy quarks, i.e. quarks whose mass $m$ is large enough $(m \gg \Lambda)$ to be considered in the perturbative domain. The top quark is certainly heavy. Also the charm and bottom quarks are usually considered to satisfy this requirement.

Heavy-quark fragmentation processes can be described by the perturbative fragmentation formalism, which resums collinearly-enhanced perturbative contributions. In the large- $x$ region, however, there are also logarithmically-enhanced contributions due to soft radiation. Proper resummation of both classes of logarithms is essential to ensure the reliability of the theoretical predictions. In the following we first review the perturbative fragmentation formalism, and then discuss soft-gluon resummation.

\subsection{Collinear resummation}

The fragmentation of heavy quarks is a collinear-safe process, because perturbative collinear singularities are regularized by the finite value of the heavy-quark mass $m$. Thus heavyquark fragmentation cross sections can unambiguously be computed order by order in QCD perturbation theory. Nonetheless, when the hard scale $Q$ of the fragmentation process is much larger than $m$, the perturbative series contains large logarithmic contributions, $\alpha_{\mathrm{S}}^{m}\left(\alpha_{\mathrm{S}} \ln Q^{2} / m^{2}\right)^{n}$, of collinear origin. These logarithmic contributions have to be resummed to higher perturbative orders, and their resummation can be performed in a process-independent way by using the perturbative fragmentation function formalism [15, 16]. The formalism amounts to generalizing the factorization formula in Eq. (1). 
The cross section $\sigma_{\mathcal{Q}}$ for the inclusive production of the heavy quark $\mathcal{Q}$ can be written as

$$
\sigma_{\mathcal{Q}}(x, Q ; m)=\sum_{a} \int_{x}^{1} \frac{d z}{z} \hat{\sigma}_{a}\left(x / z, \alpha_{\mathrm{S}}\left(\mu^{2}\right) ; Q^{2}, \mu^{2}, \mu_{F}^{2}\right) D_{a / \mathcal{Q}}\left(z, \mu_{F}^{2}, m^{2}\right)+\mathcal{O}\left((m / Q)^{p}\right)
$$

where $\hat{\sigma}_{a}(a=q, \bar{q}, g)$ are the corresponding partonic cross sections in massless QCD and $D_{a / \mathcal{Q}}$ is the pertubative fragmentation function of the (massless) parton $a$ into the heavy quark $\mathcal{Q}$. The term $\mathcal{O}\left((m / Q)^{p}\right)$ on the right-hand side stands for contributions that are suppressed by some power $p(p \geq 1)$ of $m$ in the kinematic regime $m \ll Q$. These contributions do not require all-order resummation and can be computed at a given fixed order in $\alpha_{\mathrm{S}}$. Non-perturbative corrections of the type $\Lambda / Q$ and $\Lambda / m$ are understood on the right-hand side of Eq. (38).

Note that $\sigma_{\mathcal{Q}}$ denotes a generic fragmentation cross section produced by lepton [15, 17], hadron [16, 30] or photon [31] collisions. Thus, $\hat{\sigma}_{a}$ can implicitly contain convolutions with parton distributions and fragmentation functions of light hadrons. The variable $x$ in Eq. (38) generically denotes the momentum fraction of the heavy quark ( $x$ can be either the energy fraction in $e^{+} e^{-}$annihilation or the transverse-momentum fraction in hadron and photon collisions).

The resummation of the collinear logarithms of the ratio $Q^{2} / m^{2}$ is achieved by writing the $N$ moments of the perturbative fragmentation function as

$$
D_{a / \mathcal{Q}, N}\left(\mu_{F}^{2}, m^{2}\right)=\sum_{b} E_{a b, N}\left(\mu_{F}^{2}, \mu_{0 F}^{2}\right) D_{b / \mathcal{Q}, N}^{\mathrm{ini}}\left(\alpha_{\mathrm{S}}\left(\mu_{0}^{2}\right) ; \mu_{0}^{2}, \mu_{0 F}^{2}, m^{2}\right),
$$

where $E_{a b, N}\left(\mu_{F}^{2}, \mu_{0 F}^{2}\right)$ is the evolution operator obtained by solving the AP equations (3),

$$
\frac{d E_{a b, N}\left(\mu_{F}^{2}, \mu_{0 F}^{2}\right)}{d \ln \mu_{F}^{2}}=\sum_{b} \gamma_{a b, N}\left(\alpha_{\mathrm{S}}\left(\mu_{F}^{2}\right)\right) E_{a b, N}\left(\mu_{F}^{2}, \mu_{0 F}^{2}\right)
$$

with the initial condition $E_{a b, N}\left(\mu_{0 F}^{2}, \mu_{0 F}^{2}\right)=\delta_{a b}$. The starting point of the perturbative evolution in Eq. (39) is set by the scale $\mu_{0 F}$, which has to be chosen of the same order as $m$, and by the initial condition $D_{b / \mathcal{Q}, N}^{\mathrm{ini}}$, which is perturbatively computable as power series in $\alpha_{\mathrm{S}}$ :

$$
D_{a / \mathcal{Q}, N}^{\mathrm{ini}}\left(\alpha_{\mathrm{S}}\left(\mu_{0}^{2}\right) ; \mu_{0}^{2}, \mu_{0 F}^{2}, m^{2}\right)=\delta_{a q}+\sum_{n=1}^{\infty} \alpha_{\mathrm{S}}^{n}\left(\mu_{0}^{2}\right) D_{a / \mathcal{Q}, N}^{\mathrm{ini}(n)}\left(\mu_{0}^{2}, \mu_{0 F}^{2}, m^{2}\right) .
$$

Note that, by analogy with the partonic cross sections $\hat{\sigma}_{a}$ in Eq. (38), the perturbative expansion of the initial condition $D_{a / \mathcal{Q}, N}^{\mathrm{ini}}$ depends on the factorization scale $\mu_{0 F}$ and on the renormalization scale $\mu_{0}$.

The process-dependence of the cross section $\sigma_{\mathcal{Q}}(x, Q ; m)$ is entirely embodied in the massless partonic cross sections $\hat{\sigma}_{a}$. The perturbative fragmentation function $D_{a / \mathcal{Q}, N}\left(\mu_{F}^{2}, m^{2}\right)$ (as well as $E_{a b, N}$ and $D_{a / \mathcal{Q}, N}^{\text {ini }}$ ) is instead universal (process-independent) and can be evaluated once for all. The resummation of the large collinear logarithms is obtained by perturbatively solving the AP equations in Eq. (40) and by setting $\mu_{F} \sim \mu \sim Q$ and $\mu_{0 F} \sim \mu_{0} \sim m$. For instance, we can consider the second-order expansion of the flavour non-singlet component $\gamma_{q q, N}$ of the anomalous dimensions,

$$
\gamma_{q q, N}\left(\alpha_{\mathrm{S}}\right)=\frac{\alpha_{\mathrm{S}}}{2 \pi}\left[P_{N}^{(0)}+\frac{\alpha_{\mathrm{S}}}{2 \pi} P_{N}^{(1)}+\mathcal{O}\left(\alpha_{\mathrm{S}}^{2}\right)\right]
$$


where $P_{N}^{(0)}$ and $P_{N}^{(1)}$ are the corresponding AP probabilities at LO and NLO. The evolution operator $E_{N}\left(\mu_{F}^{2}, \mu_{0 F}^{2}\right)$ for the non-singlet channel thus reads

$$
\begin{aligned}
E_{N}\left(\mu_{F}^{2}, \mu_{0 F}^{2}\right) & =\left[\frac{\alpha_{\mathrm{S}}\left(\mu_{0 F}^{2}\right)}{\alpha_{\mathrm{S}}\left(\mu_{F}^{2}\right)}\right]^{\frac{P_{N}^{(0)}}{2 \pi b_{0}}} \exp \left\{\frac{\left(\alpha_{\mathrm{S}}\left(\mu_{0 F}^{2}\right)-\alpha_{\mathrm{S}}\left(\mu_{F}^{2}\right)\right)}{4 \pi^{2} b_{0}}\left(P_{N}^{(1)}-\frac{2 \pi b_{1}}{b_{0}} P_{N}^{(0)}\right)\right. \\
& \left.+\mathcal{O}\left(\alpha_{\mathrm{S}}^{n+2} \ln ^{n} \frac{\mu_{F}^{2}}{\mu_{0 F}^{2}}\right)\right\},
\end{aligned}
$$

where the first factor on the right-hand side corresponds to the LO approximation, the first term in the curly bracket gives the NLO correction, and so forth. The leading collinear logarithms $\left(\alpha_{\mathrm{S}} \ln Q^{2} / m^{2}\right)^{n}$ of Eq. (B8) are resummed by combining the LO expression of the evolution operator with the LO evaluation of the partonic cross sections $\hat{\sigma}_{a}$ and of the initial condition $D_{a / \mathcal{Q}, N}^{\mathrm{ini}}$. The resummation of the next-to-leading collinear terms $\alpha_{\mathrm{S}}\left(\alpha_{\mathrm{S}} \ln Q^{2} / m^{2}\right)^{n}$ requires the NLO evaluation of the evolution operator, of the partonic cross sections and of the initial condition.

Although the perturbative fragmentation function is process-independent, it is not unambiguously computable. More precisely, in Eq. (38) the separation between partonic cross sections and the perturbative fragmentation function depends on the factorization scheme. Since massless partonic cross sections are usually evaluated in the $\overline{\mathrm{MS}}$ factorization scheme, in the following we always consider their definition in this scheme. This (implicitly) fixes the factorization scheme for the perturbative fragmentation function.

Since the masses of the charm and bottom quarks are not very large, these quarks typically undergo a non-perturbative fragmentation process rather than perturbatively decay into lighter partons. In this non-perturbative process, hadronization takes place, eventually producing, for instance, an observable heavy meson $H$. Heavy-hadron fragmentation cross sections can still be computed by using the factorization formula (38), provided the initial condition $D_{b / \mathcal{Q}}^{\text {ini }}$ for the perturbative fragmentation function is convoluted with a non-perturbative fragmentation distribution $D_{\mathcal{Q} / H}^{\text {np }}$ [32 34]. The most popular approach to describe $D_{\mathcal{Q} / H}^{\mathrm{np}}$ is the phenomenological model by Peterson et al. [35]. Other approaches [36, 37], based on heavy-quark effective theory, are available. The hadronization process can also be described by modelling non-perturbative effects in the perturbative fragmentation function [17, 38]. Recent studies [39] of non-perturbative effects may suggest other descriptions of $D_{\mathcal{Q} / H}^{\mathrm{np}}$, based on a shape function to be matched to the perturbative part.

At present the initial condition in Eq. (41) is known up to NLO. The calculation was first performed in Ref. [15] by computing the single inclusive cross section for heavy-quark production in $e^{+} e^{-}$annihilation and subtracting the corresponding cross section in the massless case. Using the $\overline{\mathrm{MS}}$ factorization scheme and limiting ourselves to the flavour non-singlet channel, the result is

$D^{\text {ini }}\left(x, \alpha_{\mathrm{S}}\left(\mu_{0}^{2}\right) ; \mu_{0}^{2}, \mu_{0 F}^{2}, m^{2}\right)=\delta(1-x)+\frac{\alpha_{\mathrm{S}}\left(\mu_{0}^{2}\right)}{2 \pi} C_{F}\left[\frac{1+x^{2}}{1-x}\left(\ln \frac{\mu_{0 F}^{2}}{(1-x)^{2} m^{2}}-1\right)\right]_{+}+\mathcal{O}\left(\alpha_{\mathrm{S}}^{2}\right)$,

where the plus-distribution is defined in Eq. (12). 
Considering the $N$ moments of Eq. (44) in the large- $N$ limit, we have

$$
\begin{aligned}
D_{N}^{\mathrm{ini}}\left(\alpha_{\mathrm{S}}\left(\mu_{0}^{2}\right) ; \mu_{0}^{2}, \mu_{0 F}^{2}, m^{2}\right) & =1+\frac{\alpha_{\mathrm{S}}\left(\mu_{0}^{2}\right)}{\pi} C_{F}\left[-\ln ^{2} N+\left(\ln \frac{m^{2}}{\mu_{0 F}^{2}}-2 \gamma_{E}+1\right) \ln N\right. \\
& \left.+1-\frac{\pi^{2}}{6}+\gamma_{E}-\gamma_{E}^{2}+\left(\gamma_{E}-\frac{3}{4}\right) \ln \frac{m^{2}}{\mu_{0 F}^{2}}+\mathcal{O}\left(\frac{1}{N}\right)\right]+\mathcal{O}\left(\alpha_{\mathrm{S}}^{2}\right)
\end{aligned}
$$

This expression contains logarithmic contributions proportional to $\ln ^{2} N$ and $\ln N$. After having factorized and resummed large collinear terms, $\left(\alpha_{\mathrm{S}} \ln \mu_{F}^{2} / \mu_{0 F}^{2}\right)^{n}$, by the evolution of the perturbative fragmentation function, we still have to deal with the presence of $\ln N$ terms in the initial condition. These terms spoil the convergence of the fixed-order perturbative expansion at large $N$ (or, equivalenty, at large $x$ ), and have to be resummed to all perturbative orders. Note that these terms are present independently of the analogous terms that have already been factorized at scale $\mu_{F} \sim Q$ and, possibly, resummed in the process-dependent partonic cross sections $\hat{\sigma}_{a}$ of Eq. (38). The $\ln N$ enhancement in Eq. (45) is a process-independent Sudakov effect due to soft-gluon radiation from the heavy quark at the scale $\mu_{0} \sim m$. This effect can be quantitatively more important than that in the partonic cross sections, since it is controlled by the coupling $\alpha_{\mathrm{S}}\left(\mu_{0}^{2}\right)$ that is larger than $\alpha_{\mathrm{S}}\left(\mu_{F}^{2}\right)$.

In the following, we first discuss a general method to compute the initial condition $D^{\text {ini }}$ at any order in perturbation theory. We explicitly apply the method at NLO, and we re-derive the result in Eq. (44); we then perform all-order resummation of the large-ln $N$ terms up to NLL accuracy.

\subsection{Quasi-collinear factorization and the perturbative initial con- dition}

To understand the origin of the initial condition for the perturbative fragmentation function, it is convenient to compare the heavy-quark cross section $\sigma_{\mathcal{Q}}(x, Q ; m)$ in Eq. (38) with the corresponding perturbative cross section, $\sigma_{q}(x, Q ; \epsilon)$, for the production of a massless quark $q$. The latter is collinearly divergent, and thus we regularize the divergences by working in $d=4-2 \epsilon$ space-time dimensions. In these cross sections, both the massive and massless quarks are produced at the large scale $Q$; they then perturbatively fragment by decreasing their momentum fraction $x$ and radiating some amount $q_{\perp}$ of transverse momentum. As long as $q_{\perp}$ is large, the massive quark behaves like a massless one and, thus, $d \sigma_{\mathcal{Q}} / d q_{\perp}=d \sigma_{q} / d q_{\perp}$. The difference between $\sigma_{\mathcal{Q}}$ and $\sigma_{q}$ is produced by radiation at low transverse momentum, say $\mathbf{q}_{\perp}^{2}<\mu_{0 F}^{2}$, where $\mu_{0 F}$ is an arbitrary scale such that $Q \gg \mu_{0 F}$ (and $\mu_{0 F}>m$ in the massive case). Since we are interested in the limit $m / Q \ll 1$, we can choose a value of $\mu_{0 F}$ that is parametrically very small and exploit the universal factorization properties 40] of QCD radiation at low transverse momenta to write the $N$ moments of the cross sections as

$$
\begin{aligned}
\sigma_{\mathcal{Q}, N}(Q ; m) & =\sum_{a} \widetilde{\sigma}_{a, N}\left(Q ; \mu_{0 F}\right) \widetilde{D}_{a / \mathcal{Q}, N}^{H}\left(\mu_{0 F}, m\right)+\mathcal{O}(m / Q) \\
\sigma_{q, N}(Q ; \epsilon) & =\sum_{a} \widetilde{\sigma}_{a, N}\left(Q ; \mu_{0 F}\right) \widetilde{D}_{a / q, N}^{L}\left(\mu_{0 F}, \epsilon\right)+\mathcal{O}(\epsilon)
\end{aligned}
$$


The factor $\widetilde{\sigma}$ on the right-hand side comes from the high- $q_{\perp}$ region and therefore, in the limits $m \rightarrow 0$ and $\epsilon \rightarrow 0$, it equally contributes to Eqs. (46) and (47). The fragmentation contributions $\widetilde{D}_{a / \mathcal{Q}, N}^{H}\left(\mu_{0 F}, m\right)$ and $\widetilde{D}_{a / q, N}^{L}\left(\mu_{0 F}, \epsilon\right)$, which come from the low- $q_{\perp}$ region $\left(\mathbf{q}_{\perp}^{2}<\right.$ $\left.\mu_{0 F}^{2}\right)$, are different in the heavy-quark and massless-quark cases. These contributions, which are process-independent, are divergent in the limits $m \rightarrow 0$ and $\epsilon \rightarrow 0$, respectively. In the heavy-quark case, the divergences are avoided by keeping $m$ finite, although neglecting corrections suppressed by powers of $m / \mu_{0 F}$ when $m / \mu_{0 F} \rightarrow 0$. In the massless case, the divergences are $\epsilon$-poles and have to be properly factorized to obtain the short-distance partonic cross section $\hat{\sigma}_{a, N}$ that appears in Eqs. (11) and (38). The latter is defined by

$$
\hat{\sigma}_{a, N}\left(Q, \mu_{0 F}\right)=\sum_{b} \sigma_{b, N}(Q ; \epsilon)\left[\Gamma_{N}^{(\overline{\mathrm{MS}})}\left(\mu_{0 F}, \epsilon\right)\right]_{b a}^{-1}
$$

where $\sigma_{b, N}(Q ; \epsilon)$ is the dimensionally regularized cross section in Eq. (47) and $\boldsymbol{\Gamma}$ is a matrix with respect to the flavour indices $a, b$ of the massless partons. Owing to the factorization theorem of mass singularities [四, this matrix is process-independent (though factorizationscheme-dependent) and contains all the $\epsilon$-poles that cancel the singularities of $\sigma_{b, N}(Q ; \epsilon)$ in the limit $\epsilon \rightarrow 0$. The explicit expression of $\Gamma^{(\overline{\mathrm{MS}})}$ as a function of the anomalous dimensions $\gamma_{a b, N}\left(\alpha_{\mathrm{S}}\right)$ in the $\overline{\mathrm{MS}}$ factorization scheme is given in Ref. [5].

Inserting Eq. (47) in Eq. (48), we have $\hat{\sigma}=\widetilde{\sigma} \widetilde{D}^{L} \Gamma^{-1}$. This expression for $\hat{\sigma}$ can be inserted in Eq. (38) to get $\sigma_{\mathcal{Q}}=\widetilde{\sigma} \widetilde{D}^{L} \Gamma^{-1} D^{\text {ini }}$. By comparison with Eq. (46), we thus obtain the final result

$$
\sum_{b, c} \widetilde{D}_{a / b, N}^{L}\left(\mu_{0 F}, \epsilon\right)\left[\boldsymbol{\Gamma}_{N}^{(\overline{\mathrm{MS}})}\left(\mu_{0 F}, \epsilon\right)\right]_{b c}^{-1} D_{c / \mathcal{Q}, N}^{\mathrm{ini}}\left(\alpha_{\mathrm{S}}\left(\mu_{0}^{2}\right) ; \mu_{0}^{2}, \mu_{0 F}^{2}, m^{2}\right)=\widetilde{D}_{a / \mathcal{Q}, N}^{H}\left(\mu_{0 F}, m\right) .
$$

Note that the high- $q_{\perp}$ and process-dependent contributions $\widetilde{\sigma}_{a, N}$ of Eqs. (46) and (47) do not appear in Eq. (49). The master equation (49) relates only process-independent contributions due to fragmentation at low transverse momenta, and it can be used to explicitly compute the initial condition $D_{a / \mathcal{Q}, N}^{\text {ini }}$. To this purpose we have to evaluate $\widetilde{D}^{H}$ and $\widetilde{D}^{L}$, cancel the $\epsilon$-poles in $\widetilde{D}^{L}$ by using the known expression of $\boldsymbol{\Gamma}^{(\overline{\mathrm{MS}})}$, and perform the limits $\epsilon \rightarrow 0$ and $m / \mu_{0 F} \rightarrow 0$.

The factorization of the low- $q_{\perp}$ contributions $\widetilde{D}^{H}$ and $\widetilde{D}^{L}$ in Eqs. (46) and (47) follows from the corresponding factorization formulae (Fig. 5) of the QCD matrix elements (evaluated in physical gauges). As is well known [5, 22, 23, 40, in the case of massless QCD the matrix element factorization for the splitting process $a \rightarrow q\left(p_{1}\right)+X\left(\left\{q_{i}\right\}\right)(X$ denotes a set of partons with momenta $q_{i}$ and total momentum $\left.q=\sum_{i} q_{i}\right)$ is controlled by the collinear limit. As emphasized in Ref. 41, the dynamics of the splitting processes of massive partons is described by analogous factorization formulae, which are obtained by generalizing the collinear limit to the quasi-collinear limit. Considering the splitting process $a \rightarrow \mathcal{Q}\left(p_{1}\right)+X\left(\left\{q_{i}\right\}\right), \mathcal{Q}$ being a heavy quark of mass $m$ and momentum $p_{1}^{\nu}\left(p_{1}^{2}=m^{2}\right)$, we can describe its kinematics in terms of the following Sudakov parametrization:

$$
p_{1}^{\nu}=z p^{\nu}-q_{\perp}^{\nu}+\frac{\mathbf{q}_{\perp}^{2}+\left(1-z^{2}\right) m^{2}}{z} \frac{n^{\nu}}{2 p \cdot n},
$$

\footnotetext{
${ }^{\ddagger}$ To simplify the notation, we set $\mu=\mu_{F}=\mu_{0 F}$ in Eq. (38).
} 


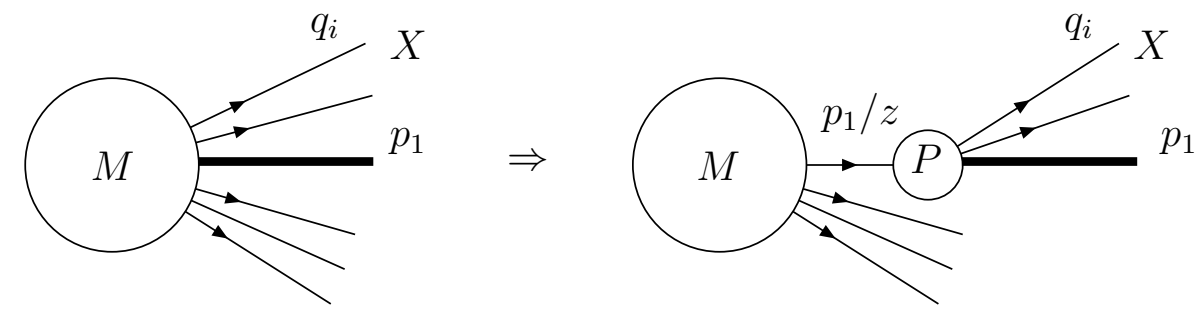

Figure 5: Schematic picture of matrix element factorization in the collinear and quasi-collinear limits. The thicker line denotes the particle (massless parton or heavy quark) that undergoes the collinear or quasi-collinear splitting process.

$$
q^{\nu}=(1-z) p^{\nu}+q_{\perp}^{\nu}+\frac{q^{2}+\mathbf{q}_{\perp}^{2}-(1-z)^{2} m^{2}}{1-z} \frac{n^{\nu}}{2 p \cdot n}
$$

where the momentum $p^{\nu}$ (with $p^{2}=m^{2}$ ) denotes the collinear ('forward') direction, and the light-like vector $n^{\nu}\left(n^{2}=0\right)$ denotes the 'backward' direction $\left(q_{\perp} \cdot p=q_{\perp} \cdot n=0, \mathbf{q}_{\perp}^{2}=\right.$ $\left.-q_{\perp}^{\nu} q_{\perp \nu}\right)$. The customary Sudakov parametrization for the splitting process of a massless quark is recovered from Eq. (50) by simply setting $m=0$. We can now consider the collinear or quasi-collinear limits. In the massless case the collinear region is defined by the limit $q_{\perp} \rightarrow 0$. In the heavy-quark case the quasi-collinear region is defined 41] by the limits $q_{\perp} \rightarrow 0$ and $m \rightarrow 0$ at fixed ratio $m^{2} / \mathbf{q}_{\perp}^{2}$. Note that the key difference between the collinear and quasi-collinear limits is that the latter has to be performed by keeping the mass $m$ of the same order as $q_{\perp}$ while $q_{\perp} \rightarrow 0$.

Performing the calculation of the collinear or quasi-collinear limits, a generic QCD matrix element $M\left(p_{1},\left\{q_{i}\right\} ; \ldots\right)$ factorizes in terms of a process-dependent contribution (which is finite in the limiting region) and a universal AP splitting function [22, 23, 41]. The fragmentation contributions $\widetilde{D}^{L}$ and $\widetilde{D}^{H}$ in Eq. (49) are obtained by integrating the AP splitting functions over the momenta $q_{i}$ of the partons involved in the corresponding splitting process.

In this section we have so far outlined a general method to compute the initial condition $D^{\text {ini }}$ for heavy-quark fragmentation. As an example, we now apply the method to the explicit calculation at NLO of the flavour non-singlet component of $D^{\text {ini }}$.

To this purpose, we have to consider the splitting process $\mathcal{Q} \rightarrow \mathcal{Q}\left(p_{1}\right)+g(q)$ at $\mathcal{O}\left(\alpha_{\mathrm{S}}\right)$. Performing the collinear or quasi-collinear limits, the matrix element $M\left(p_{1}, q ; \ldots\right)$ factorizes as (see Fig. 5)

$$
\left|M\left(p_{1}, q ; \ldots\right)\right|^{2} \simeq\left|M\left(p_{1} / z ; \ldots\right)\right|^{2} \frac{4 \pi \alpha_{\mathrm{S}}}{p_{1} \cdot q} \hat{P}=\left|M\left(p_{1} / z ; \ldots\right)\right|^{2} 8 \pi \alpha_{\mathrm{S}} \frac{z(1-z)}{\mathbf{q}_{\perp}^{2}+(1-z)^{2} m^{2}} \hat{P}
$$

where we have used the parametrization in Eq. (50), the mass-shell condition $q^{2}=0$ and the identity

$$
2 p_{1} \cdot q=\frac{\mathbf{q}_{\perp}^{2}+(1-z)^{2} m^{2}}{z(1-z)} .
$$


In the massless case, $\hat{P}$ is the usual AP splitting function in $d=4-2 \epsilon$ dimensions,

$$
\hat{P}_{q g}(z ; \epsilon)=C_{F}\left[\frac{1+z^{2}}{1-z}-\epsilon(1-z)\right]
$$

while its generalization to the heavy-quark case in $d=4$ dimensions is

$$
\hat{P}_{\mathcal{Q} g}\left(z ; m^{2} / \mathbf{q}_{\perp}^{2}\right)=C_{F}\left[\frac{1+z^{2}}{1-z}-\frac{m^{2}}{p_{1} \cdot q}\right]=C_{F}\left[\frac{1+z^{2}}{1-z}-\frac{2 z(1-z) m^{2}}{\mathbf{q}_{\perp}^{2}+(1-z)^{2} m^{2}}\right] .
$$

To extract the low- $q_{\perp}$ contributions $\widetilde{D}^{L}$ and $\widetilde{D}^{H}$ in Eq. (49), we consider the $N$ moments of the factorization formula (51) and perform the integration of the AP splitting functions in Eqs. (53) and (54) over the low transverse-momentum region, $\mathbf{q}_{\perp}^{2}<\mu_{0 F}^{2}$, of the radiated gluon. The phase space for gluon radiation at fixed value of the longitudinal momentum $p_{1} \cdot n$ of the triggered quark is

$$
d \Phi=\left(\mu_{r}\right)^{4-d} \frac{d^{d} q}{(2 \pi)^{d-1}} \delta_{+}\left(q^{2}\right)=\frac{1}{16 \pi^{2}} \frac{1}{\Gamma(1-\epsilon)} d \mathbf{q}_{\perp}^{2}\left(\frac{4 \pi \mu_{r}^{2}}{\mathbf{q}_{\perp}^{2}}\right)^{\epsilon} \frac{d z}{z(1-z)} \Theta(z(1-z))
$$

where $\mu_{r}$ is the dimensional-regularization scale. We thus obtain

$$
\begin{aligned}
\widetilde{D}_{N}^{L}\left(\mu_{0 F}, \epsilon\right) & =1+\frac{\alpha_{\mathrm{S}}}{2 \pi} \frac{1}{\Gamma(1-\epsilon)} \int_{0}^{\mu_{0 F}^{2}} d \mathbf{q}_{\perp}^{2}\left(\frac{4 \pi \mu_{r}^{2}}{\mathbf{q}_{\perp}^{2}}\right)^{\epsilon} \int_{0}^{1} d z\left(z^{N-1}-1\right) \frac{1}{\mathbf{q}_{\perp}^{2}} \hat{P}_{q g}(z ; \epsilon) \\
& =1-\frac{\alpha_{\mathrm{S}}}{2 \pi} \frac{1}{\Gamma(1-\epsilon)} \frac{1}{\epsilon}\left(\frac{4 \pi \mu_{r}^{2}}{\mu_{0 F}^{2}}\right)^{\epsilon} \int_{0}^{1} d z\left(z^{N-1}-1\right) C_{F}\left[\frac{1+z^{2}}{1-z}-\epsilon(1-z)\right]
\end{aligned}
$$

and

$$
\begin{aligned}
& \widetilde{D}_{N}^{H}\left(\mu_{0 F}, m\right)=1+\frac{\alpha_{\mathrm{S}}}{2 \pi} \int_{0}^{\mu_{0 F}^{2}} d \mathbf{q}_{\perp}^{2} \int_{0}^{1} d z\left(z^{N-1}-1\right) \frac{1}{\mathbf{q}_{\perp}^{2}+(1-z)^{2} m^{2}} \hat{P}_{\mathcal{Q} g}\left(z ; m^{2} / \mathbf{q}_{\perp}^{2}\right) \\
& =1+\frac{\alpha_{\mathrm{S}}}{2 \pi} C_{F} \int_{0}^{1} d z\left(z^{N-1}-1\right)\left[\frac{1+z^{2}}{1-z} \ln \frac{\mu_{0 F}^{2}+(1-z)^{2} m^{2}}{(1-z)^{2} m^{2}}-\frac{2 z}{(1-z)} \frac{\mu_{0 F}^{2}}{\mu_{0 F}^{2}+(1-z)^{2} m^{2}}\right] \\
& =1+\frac{\alpha_{\mathrm{S}}}{2 \pi} C_{F} \int_{0}^{1} d z\left(z^{N-1}-1\right)\left[\frac{1+z^{2}}{1-z} \ln \frac{\mu_{0 F}^{2}}{(1-z)^{2} m^{2}}-\frac{2 z}{(1-z)}\right]+\mathcal{O}\left(m^{2} / \mu_{0 F}^{2}\right) .
\end{aligned}
$$

Note that the massless-quark calculation in Eq. (56) has consistently been performed by using dimensional regularization, while in the heavy-quark calculation of Eq. (58) we have set $d=4$. The weight factor $\left(z^{N-1}-1\right)$ in Eqs. (56) and (58) takes into account real $\left({ }^{N-1}\right)$ and virtual ('-1') gluon radiation. The real contribution derives directly from Eq. (51). The virtual contribution has been included by simply enforcing the constraint $\widetilde{D}_{N=1}^{L}=\widetilde{D}_{N=1}^{H}=1$, which follows from the conservation of the fermion number in the non-singlet sector.

The initial condition $D_{N}^{\text {ini }}$ is obtained from the master equation (49), which in the nonsinglet sector simplifies as

$$
D_{N}^{\mathrm{ini}}\left(\alpha_{\mathrm{S}}\left(\mu_{0}^{2}\right) ; \mu_{0}^{2}, \mu_{0 F}^{2}, m^{2}\right)=\frac{\widetilde{D}_{N}^{H}\left(\mu_{0 F}, m\right)}{\widetilde{D}_{N}^{L}\left(\mu_{0 F}, \epsilon\right)\left[\Gamma_{N}^{(\overline{\mathrm{MS}})}\left(\mu_{0 F}, \epsilon\right)\right]^{-1}},
$$


or, equivalently, at $\mathcal{O}\left(\alpha_{\mathrm{S}}\right)$ :

$$
D_{N}^{\mathrm{ini}}\left(\alpha_{\mathrm{S}}\left(\mu_{0}^{2}\right) ; \mu_{0}^{2}, \mu_{0 F}^{2}, m^{2}\right)=1+\left\{\widetilde{D}_{N}^{H}\left(\mu_{0 F}, m\right)-\widetilde{D}_{N}^{L}\left(\mu_{0 F}, \epsilon\right)\left[\Gamma_{N}^{(\overline{\mathrm{MS}})}\left(\mu_{0 F}, \epsilon\right)\right]^{-1}\right\}+\mathcal{O}\left(\alpha_{\mathrm{S}}^{2}\right) .
$$

To evaluate the massless-quark contribution $\widetilde{D}^{L} \Gamma^{-1}$, we use the $\mathcal{O}\left(\alpha_{\mathrm{S}}\right)$ expression of the collinear counterterm $\Gamma_{N}^{(\overline{\mathrm{MS}})}$ in the $\overline{\mathrm{MS}}$ factorization scheme [5]:

$$
\Gamma_{N}^{(\overline{\mathrm{MS}})}\left(\mu_{0 F}, \epsilon\right)=1-\frac{\alpha_{\mathrm{S}}}{2 \pi} \frac{1}{\Gamma(1-\epsilon)} \frac{1}{\epsilon}\left(\frac{4 \pi \mu_{r}^{2}}{\mu_{0 F}^{2}}\right)^{\epsilon} \int_{0}^{1} d z\left(z^{N-1}-1\right) C_{F} \frac{1+z^{2}}{1-z}+\mathcal{O}\left(\alpha_{\mathrm{S}}^{2}\right) .
$$

Using Eqs. (56) and (61) we obtain

$$
\widetilde{D}_{N}^{L}\left(\mu_{0 F}, \epsilon\right)\left[\Gamma_{N}^{(\overline{\mathrm{MS}})}\left(\mu_{0 F}, \epsilon\right)\right]^{-1}=1+\frac{\alpha_{\mathrm{S}}}{2 \pi} C_{F} \int_{0}^{1} d z\left(z^{N-1}-1\right)[1-z]+\mathcal{O}(\epsilon) .
$$

Note that this expression contains no $\epsilon$ poles and the dependence on the dimensionalregularization scale $\mu_{r}$ has consistently disappeared in the limit $\epsilon \rightarrow 0$. Note also that the finite (when $\epsilon \rightarrow 0$ ) term on the right-hand side entirely derives from the $\epsilon$-dependent part of the AP splitting function in Eq. (53).

Inserting Eq. (62) in Eq. (60) and using Eq. (58), we finally obtain the $\mathcal{O}\left(\alpha_{\mathrm{S}}\right)$ contribution to the initial condition:

$$
\begin{aligned}
D_{N}^{\mathrm{ini}}\left(\alpha_{\mathrm{S}}\left(\mu_{0}^{2}\right) ; \mu_{0}^{2}, \mu_{0 F}^{2}, m^{2}\right) & =1+\frac{\alpha_{\mathrm{S}}}{2 \pi} C_{F} \int_{0}^{1} d z\left(z^{N-1}-1\right)\left[\frac{1+z^{2}}{1-z}\left(\ln \frac{\mu_{0 F}^{2}}{(1-z)^{2} m^{2}}-1\right)\right] \\
& +\mathcal{O}\left(\alpha_{\mathrm{S}}^{2}\right) .
\end{aligned}
$$

This result agrees with the known [15] expression in Eq. (44). Our calculation explicitly shows that the plus-distribution in Eq. (44) receives two contributions: the term in the square bracket of Eq. (58) and that in the square bracket of Eq. (62). The former is due to heavy-quark fragmentation at low $q_{\perp}$, while the latter entirely depends on the prescription (dimensional regularization and $\overline{\mathrm{MS}}$ factorization) used to handle the collinear divergences in the fragmentation of the massless quark. A derivation of Eq. (44) similar to ours is presented in Ref. [42].

\subsection{Soft-gluon resummation for the initial condition}

The $\mathcal{O}\left(\alpha_{\mathrm{S}}\right)$ calculation presented in the second part of Sect. 3.2 can be extended to the flavour singlet components of $D_{a / \mathcal{O}}^{\mathrm{ini}}$. The method discussed in the first part of Sect. 3.2 can also be used to evaluate $D^{\text {ini }}$ at $\mathcal{O}\left(\alpha_{\mathrm{S}}^{2}\right)$ (and at higher orders), provided the calculation of the collinear (massless) factorization formulae of Refs. [22, 23] is generalized to the quasicollinear (heavy-quark) case. In this section we use the master equation (49) (or Eq. (59)) to perform all-order resummation of the soft-gluon contributions to $D_{N}^{\text {ini }}$ at large $N$ (or, equivalently, at large $x$ ). We limit ourselves to considering the non-singlet component, since the flavour-singlet contributions are suppressed by a relative factor of $\mathcal{O}(1 / N)$ when $N \rightarrow \infty$. 
At high perturbative orders the fragmentation factors $\widetilde{D}_{N}^{H}$ and $\widetilde{D}_{N}^{L}$ receive contributions from multiple radiation of partons with momenta $q_{i}$. All-order resummation of these contributions is achieved as in the case of the customary AP evolution in transverse momentum. The region of ordered transverse momenta, $\mu_{0 F}^{2}>\ldots>\mathbf{q}_{\perp i}^{2}>\mathbf{q}_{\perp j}^{2}>\ldots>0$, leads to the exponentiation of the lowest-order AP splitting kernel. Then, the subregions where two or more transverse momenta are of the same order $\left(\mathbf{q}_{\perp_{i}}^{2} \sim \ldots \sim \mathbf{q}_{\perp j}^{2}\right)$ lead to perturbative corrections to the exponentiated kernel. In particular, in the case of soft-gluon radiation the inclusive correction to the lowest-order AP kernel can be taken into account by the simple replacement [7, 9]

$$
\alpha_{\mathrm{S}} \rightarrow \alpha_{\mathrm{S}}\left(\mathbf{q}_{\perp}^{2}\right)\left[1+\frac{\alpha_{\mathrm{S}}\left(\mathbf{q}_{\perp}^{2}\right)}{2 \pi}(K+\mathcal{O}(\epsilon))+\mathcal{O}\left(\alpha_{\mathrm{S}}^{2}\right)\right]
$$

where the coefficient $K$ is given in Eq. (的), and the term $\mathcal{O}\left(\alpha_{\mathrm{S}}^{2}\right)$ only contributes beyond NLL accuracy at large $N$. This resummation procedure applies to all the factors, $\widetilde{D}_{N}^{L}, \Gamma_{N}^{(\overline{\mathrm{MS}})}$ and $\widetilde{D}_{N}^{H}$, in Eq. (59).

We first consider the case of massless-quark fragmentation. We note from Eq. (62) that the factor $\widetilde{D}_{N}^{L}\left[\Gamma_{N}^{(\overline{\mathrm{MS}})}\right]^{-1}$ does not show any $\ln N$ enhancement at $\mathcal{O}\left(\alpha_{\mathrm{S}}\right)$. Soft-gluon radiation produces $\ln N$ corrections in both Eqs. (56) and (61), but they cancel each other. Owing to Eq. (64), this cancellation mechanism is valid up to NLL order in the $\overline{\mathrm{MS}}$ factorization scheme, since both factors $\widetilde{D}_{N}^{L}\left(\mu_{0 F}, \epsilon\right)$ and $\Gamma_{N}^{(\overline{\mathrm{MS}})}\left(\mu_{0 F}, \epsilon\right)$ include soft-gluon emission up to the transverse-momentum scale $\mathbf{q}_{\perp}^{2}=\mu_{0 F}^{2}$. We thus have

$$
\widetilde{D}_{N}^{L}\left(\mu_{0 F}, \epsilon\right)\left[\Gamma_{N}^{(\overline{\mathrm{MS}})}\left(\mu_{0 F}, \epsilon\right)\right]^{-1}=1+\mathcal{O}\left(\alpha_{\mathrm{S}}\left(\alpha_{\mathrm{S}} \ln N\right)^{k}\right)
$$

In the heavy-quark case, we first exponentiate Eq. (57) and then perform the replacement of Eq. (64). We obtain

$$
\begin{aligned}
\ln \widetilde{D}_{N}^{H}\left(\mu_{0 F}, m\right)= & \int_{0}^{1} d z\left(z^{N-1}-1\right) \int_{0}^{\mu_{0 F}^{2}} \frac{d \mathbf{q}_{\perp}^{2}}{\mathbf{q}_{\perp}^{2}+(1-z)^{2} m^{2}} \\
& \cdot \frac{\alpha_{\mathrm{S}}\left(\mathbf{q}_{\perp}^{2}\right)}{2 \pi}\left[1+\frac{\alpha_{\mathrm{S}}\left(\mathbf{q}_{\perp}^{2}\right)}{2 \pi} K\right] \hat{P}_{\mathcal{Q} g}\left(z ; m^{2} / \mathbf{q}_{\perp}^{2}\right)+\mathcal{O}\left(\alpha_{\mathrm{S}}\left(\alpha_{\mathrm{S}} \ln N\right)^{k}\right)
\end{aligned}
$$

The right-hand side can be further simplified by neglecting contributions of $\mathcal{O}\left(\mathrm{m}^{2} / \mu_{0 F}^{2}\right)$ and terms beyond NLL order at large $N$. To this purpose, we note that $\hat{P}_{\mathcal{Q} g}$ contains two different contributions. In the limit $m \rightarrow 0$, the $\mathbf{q}_{\perp}^{2}$-integrals of the first and second contributions in the square bracket of Eq. (54) are respectively logarithmically and linearly divergent at small $\mathbf{q}_{\perp}^{2}$. To the required accuracy, the logarithmically-divergent and linearlydivergent integrals are dominated by the transverse-momentum regions $\mu_{0 F}^{2}>\mathbf{q}_{\perp}^{2}>(1-$ $z)^{2} m^{2}$ and $\mathbf{q}_{\perp}^{2} \simeq(1-z)^{2} m^{2}$, respectively. We thus obtain

$$
\begin{aligned}
\ln \widetilde{D}_{N}^{H}\left(\mu_{0 F}, m\right) & =\int_{0}^{1} d z \frac{z^{N-1}-1}{1-z}\left\{\int_{(1-z)^{2} m^{2}}^{\mu_{0 F}^{2}} \frac{d \mathbf{q}_{\perp}^{2}}{\mathbf{q}_{\perp}^{2}} \frac{\alpha_{\mathrm{S}}\left(\mathbf{q}_{\perp}^{2}\right)}{\pi}\left[1+\frac{\alpha_{\mathrm{S}}\left(\mathbf{q}_{\perp}^{2}\right)}{2 \pi} K\right]\right. \\
& \left.-\frac{C_{F}}{\pi} \alpha_{\mathrm{S}}\left((1-z)^{2} m^{2}\right)\right\}+\mathcal{O}\left(\alpha_{\mathrm{S}}\left(\alpha_{\mathrm{S}} \ln N\right)^{k}\right) .
\end{aligned}
$$


The expression in the curly bracket clearly shows the presence of two characteristic transversemomentum scales: the factorization scale $\mu_{0 F}^{2}$ and the heavy-quark scale $(1-z)^{2} m^{2}$. The latter is related to the angular size $\theta_{0}=m / E_{\mathcal{Q}}\left(E_{\mathcal{Q}}\right.$ is the energy of the heavy quark $)$ of the 'dead cone' [17] for bremsstrahlung off a massive particle. Writing the transverse momentum as $\mathbf{q}_{\perp}^{2} \simeq(1-z)^{2} E_{\mathcal{Q}}^{2} \theta^{2}$, where $(1-z) E_{\mathcal{Q}}$ is the energy of the radiated gluon and $\theta$ is its emission angle, the first term in the curly bracket of Eq. (67) describes soft and collinear radiation outside the dead cone $\left(\theta^{2}>\theta_{0}^{2}\right)$, while the second term is related to soft radiation near the dead-cone boundary $\left(\theta^{2} \simeq \theta_{0}^{2}\right)$.

Inserting Eq. (65) in Eq. (59), we obtain the following resummed expression for the initial condition of the heavy-quark fragmentation function:

$$
\ln D_{N}^{\text {ini }}\left(\alpha_{\mathrm{S}}\left(\mu_{0}^{2}\right) ; \mu_{0}^{2}, \mu_{0 F}^{2}, m^{2}\right)=\ln \widetilde{D}_{N}^{H}\left(\mu_{0 F}, m\right)+\mathcal{O}\left(\alpha_{\mathrm{S}}\left(\alpha_{\mathrm{S}} \ln N\right)^{k}\right)
$$

where $\ln \widetilde{D}_{N}^{H}$ is given in Eq. (67) up to NLL accuracy. Moreover, the derivation of Eqs. (65) and (67) and the dependence on the factorization scale $\mu_{0 F}$ of the perturbative fragmentation function in Eq. (39) suggest a generalization of the NLL result in Eq. (68) to any logarithmic order as follows:

$$
\begin{aligned}
\ln D_{N}^{\text {ini }}\left(\alpha_{\mathrm{S}}\left(\mu_{0}^{2}\right) ; \mu_{0}^{2}, \mu_{0 F}^{2}, m^{2}\right) & =\int_{0}^{1} d z \frac{z^{N-1}-1}{1-z}\left\{\int_{(1-z)^{2} m^{2}}^{\mu_{0 F}^{2}} \frac{d \mathbf{q}_{\perp}^{2}}{\mathbf{q}_{\perp}^{2}} A\left[\alpha_{\mathrm{S}}\left(\mathbf{q}_{\perp}^{2}\right)\right]\right. \\
& \left.+H\left[\alpha_{\mathrm{S}}\left((1-z)^{2} m^{2}\right)\right]\right\}+\mathcal{O}(1 / N) .
\end{aligned}
$$

The function $A\left(\alpha_{\mathrm{S}}\right)$, whose perturbative expansion is given in Eq. (17), is the processindependent function that controls the large- $N$ behaviour of the flavour non-singlet anomalous dimensions (see Eq. (22)). The function $H\left(\alpha_{\mathrm{S}}\right)$,

$$
H\left(\alpha_{\mathrm{S}}\right)=\sum_{n=1}^{\infty}\left(\frac{\alpha_{\mathrm{S}}}{\pi}\right)^{n} H^{(n)}
$$

is strictly related to soft radiation off a heavy quark, and its first-order coefficient is

$$
H^{(1)}=-C_{F} .
$$

The coefficient $A^{(1)}$ controls the resummation of the LL terms $\alpha_{\mathrm{S}}^{n} \ln ^{n+1} N$ in Eq. (69). The coefficients $A^{(2)}$ and $H^{(1)}$ give the NLL terms $\alpha_{\mathrm{S}}^{n} \ln ^{n} N$, and so forth. At LL accuracy, Eq. (69) agrees with the resummed calculation of Ref. [15. Knowing the coefficients $A^{(2)}$ and $H^{(1)}$ in Eqs. (19) and (71), we have explicitly extended the result of Ref. [15] up to NLL accuracy.

To quantitatively study the effect of soft-gluon resummation, we proceed as in Sect. 2.2. The Sudakov-resummed part $D_{N}^{\text {ini, } S}$ of the initial condition is written as

$$
\begin{aligned}
D_{N}^{\text {ini, } S}\left(\alpha_{\mathrm{S}}\left(\mu_{0}^{2}\right) ; \mu_{0}^{2}, \mu_{0 F}^{2}, m^{2}\right) & =\left\{1+\frac{\alpha_{\mathrm{S}}\left(\mu_{0}^{2}\right) C_{F}}{\pi}\left[-\frac{\pi^{2}}{6}+1-\gamma_{E}^{2}+\gamma_{E}+\left(\frac{3}{4}-\gamma_{E}\right) \ln \frac{\mu_{0 F}^{2}}{m^{2}}\right]\right\} \\
& \times \exp \left[\ln N g_{\text {ini }}^{(1)}\left(\lambda_{0}\right)+g_{\text {ini }}^{(2)}\left(\lambda_{0}, m^{2} / \mu_{0}^{2} ; m^{2} / \mu_{0 F}^{2}\right)\right]
\end{aligned}
$$

where

$$
\lambda_{0}=b_{0} \alpha_{\mathrm{S}}\left(\mu_{0}^{2}\right) \ln N
$$


and we have explicitly introduced the renormalization scale $\mu_{0}$, which, in general, is different from the factorization scale $\mu_{0 F}$. The LL and NLL functions $g_{\text {ini }}^{(1)}$ and $g_{\text {ini }}^{(2)}$ are obtained by performing the $z$ and $\mathbf{q}_{\perp}^{2}$ integrations of Eqs. (69) up to NLL accuracy:

$$
\begin{aligned}
g_{\text {ini }}^{(1)}\left(\lambda_{0}\right)= & -\frac{A^{(1)}}{2 \pi b_{0} \lambda_{0}}\left[2 \lambda_{0}+\left(1-2 \lambda_{0}\right) \ln \left(1-2 \lambda_{0}\right)\right], \\
g_{\text {ini }}^{(2)}\left(\lambda_{0}, m^{2} / \mu_{0}^{2} ; m^{2} / \mu_{0 F}^{2}\right)= & \frac{A^{(1)}}{2 \pi b_{0}}\left(\ln \frac{\mu_{0 F}^{2}}{m^{2}}+2 \gamma_{E}\right) \ln \left(1-2 \lambda_{0}\right) \\
& -\frac{A^{(1)} b_{1}}{4 \pi b_{0}^{3}}\left[4 \lambda_{0}+2 \ln \left(1-2 \lambda_{0}\right)+\ln ^{2}\left(1-2 \lambda_{0}\right)\right] \\
& +\frac{1}{2 \pi b_{0}}\left[2 \lambda_{0}+\ln \left(1-2 \lambda_{0}\right)\right]\left(\frac{A^{(2)}}{\pi b_{0}}+A^{(1)} \ln \frac{\mu_{0}^{2}}{\mu_{0 F}^{2}}\right) \\
& +\frac{H^{(1)}}{2 \pi b_{0}} \ln \left(1-2 \lambda_{0}\right) .
\end{aligned}
$$

Analogously to Eq. (33), the terms in the curly bracket of Eq. (72) are the constant (when $N \rightarrow \infty)$ contributions to the NLO initial condition in Eq. (45).

The final NLO+NLL resummed expression for the $N$ moments of the (non-singlet) initial condition is then given by

$$
\begin{aligned}
D_{N}^{\text {ini(res })}\left(\alpha_{\mathrm{S}}\left(\mu_{0}^{2}\right) ; \mu_{0}^{2}, \mu_{0 F}^{2}, m^{2}\right) & =D_{N}^{\mathrm{ini}, S}\left(\alpha_{\mathrm{S}}\left(\mu_{0}^{2}\right) ; \mu_{0}^{2}, \mu_{0 F}^{2}, m^{2}\right)-\left[D_{N}^{\mathrm{ini}, S}\left(\alpha_{\mathrm{S}}\left(\mu_{0}^{2}\right) ; \mu_{0}^{2}, \mu_{0 F}^{2}, m^{2}\right)\right]_{\alpha_{\mathrm{S}}} \\
& +\left[D_{N}^{\mathrm{ini}}\left(\alpha_{\mathrm{S}}\left(\mu_{0}^{2}\right) ; \mu_{0}^{2}, \mu_{0 F}^{2}, m^{2}\right)\right]_{\alpha_{\mathrm{S}}}
\end{aligned}
$$

where $\left[D_{N}^{\text {ini }}\right]_{\alpha_{\mathrm{S}}}$ are the $N$ moments of the $\mathcal{O}\left(\alpha_{\mathrm{S}}\right)$ result in Eq. (44), $D_{N}^{\text {ini,S }}$ is given in Eq. $(\square 2)$ and $\left[D_{N}^{\mathrm{ini}, S}\right]_{\alpha_{\mathrm{S}}}$ is its perturbative truncation at $\mathcal{O}\left(\alpha_{\mathrm{S}}\right)$.

Note that, analogously to Eq. (33), the Sudakov-resummed part $D_{N}^{\mathrm{ini}, S}$ of the heavyquark initial condition has cut singularities in the complex variable $N$. In the heavy-quark case the singularities start at the branch-point $N=\exp \left\{1 / 2 b_{0} \alpha_{\mathrm{S}}\left(\mu_{0}^{2}\right)\right\}$ (i.e. at $\lambda_{0}=1 / 2$ in Eqs. (74) and (75)). They signal the dominance of non-perturbative effects at very large values of $N$ (or very large $x$ ). As in the massless case, we do not explicitly include any non-perturbative contributions in our calculations for heavy-quark fragmentation, and we simply apply the Minimal Prescription of Ref. 28] when performing numerical inversions of Mellin moments to $x$ space.

The NLO and NLO+NLL calculations are compared in Fig. 6. In both calculations we consider the perturbative fragmentation function $D\left(x ; \mu_{F}^{2}, m^{2}\right)$, which is obtained from the corresponding initial condition $D^{\text {ini }}$ at the scale $\mu_{0 F}=\mu_{0}=m$ (the dependence on the scales $\mu_{0 F}$ and $\mu_{0}$ is studied in Sect. 3.4) by AP evolution (see Eqs. (39) and (43)) up to the scale $\mu_{F}$. We can see that the NLO+NLL resummed fragmentation function is much softer than the NLO fragmentation function. Moreover, in both cases, the AP evolution reduces the heavy-quark momentum, and the fragmentation function is softened.

A typical feature of all-order soft-gluon resummation is the presence of a characteristic maximum (Sudakov peak) [17 at $x=x_{\text {peak }}$ in the NLO+NLL fragmentation function. The position of the peak is at $1-x_{\text {peak }} \sim(\Lambda / m)^{c}$, where the power $c=1-\exp \left\{-\pi b_{0} / A^{(1)}\right\}$ is slightly smaller than unity. 


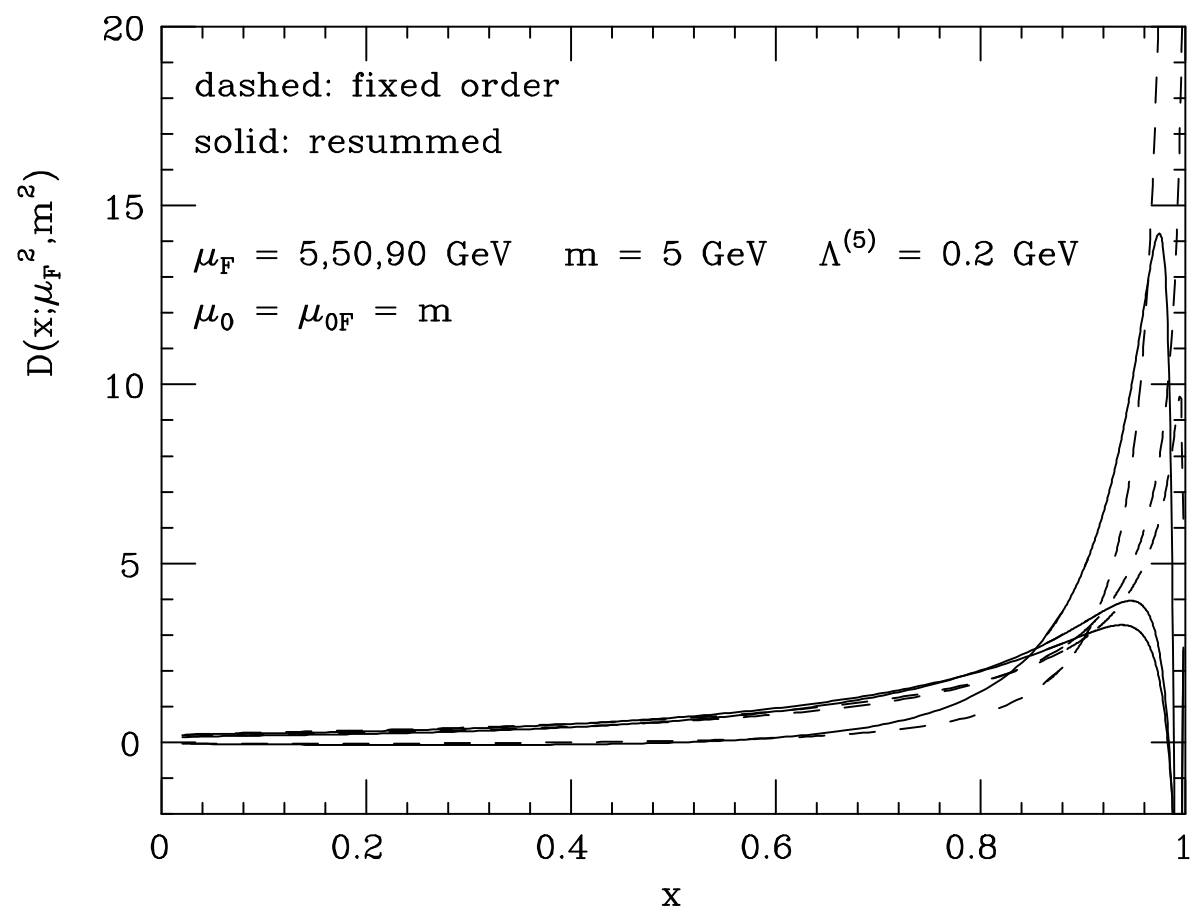

Figure 6: Effect of Sudakov resummation for the initial condition of the heavy-quark fragmentation function, evolved up to three different scales $\mu_{F}=5,50,90 \mathrm{GeV}$.

Figure 6 also shows that the fragmentation function becomes negative when $x$ is very close to $x=1$. This occurs both in the NLO+NLL calculation and in the NLO one (see also Fig. 9), although in the NLO case the effect is not very evident from Fig. 6 because it is due to a negative $\delta$-function contribution concentrated near $x=1$ (see Eq. (44)). In both cases the negative behaviour is a consequence of the approximate character of the calculations for the initial condition and, therefore, it is unphysical. Note, however, that the origin of this behaviour is different in the two cases. In the NLO case the negative behaviour is due to the presence of large, unresummed double-logarithmic terms $\alpha_{\mathrm{S}} \ln ^{2}(1-x)$. In the NLO+NLL case this pathological behaviour is cured by soft-gluon resummation, but the sensitivity to non-perturbative QCD phenomena still drives the fragmentation function negative. As a matter of fact, if we had considered soft-photon resummation for the fragmentation of a massive lepton in QED, the ensuing NLO+NLL fragmentation function would have been positive. In QCD, instead, the Landau singularity of the perturbative coupling at small transverse momenta leads to a branch-point in the resummed expression (72). The branchpoint occurs when $2 b_{0} \alpha_{\mathrm{S}}\left(m^{2}\right) \ln N=1$ in the $N$-plane and produces the negative behaviour of $D\left(x ; \mu_{F}^{2}, m^{2}\right)$ at $1-x \sim \Lambda / m$. At such large values of $x$, non-perturbative contributions have to properly be included in the evaluation of the heavy-quark fragmentation function [17, 38.

Soft-gluon resummation therefore suggests that non-perturbative fragmentation phenomena become dominant when $1-x \sim \Lambda / m$. This suggestion is consistent with the findings of the approach [36, 37] based on heavy-quark effective theory. However, the expectation of the authors of Ref. [36] that Sudakov effects are large only when $1-x \sim(\Lambda / m)^{2}$, and likely to be less important than non-perturbative effects, is not correct. In fact, the 
resummed calculation shows that the Sudakov effects are large at smaller values of $x$. In particular, the Sudakov peak is placed at $1-x_{\text {peak }} \sim(\Lambda / m)^{c}>\Lambda / m$. Around the region of the Sudakov peak, perturbative soft-gluon resummation is (at least) as important as non-perturbative effects.

The results presented in this section regard the process-independent fragmentation function $D\left(x ; \mu_{F}^{2}, m^{2}\right)$. This universal (though factorization-scheme-dependent) heavy-quark distribution enters the perturbative QCD predictions of any process-dependent cross sections for heavy-quark fragmentation (see Eq. (38)). In the next section, we explicitly consider heavy-quark fragmentation in $e^{+} e^{-}$annihilation.

\subsection{Single-inclusive heavy-quark distribution in $e^{+} e^{-}$annihilation}

The most accurate data on the fragmentation of bottom and charm quarks come from $e^{+} e^{-}$ annihilation experiments. These data can be used to test perturbative QCD predictions and to extract information on the non-perturbative contribution to heavy-quark fragmentation.

We consider the inclusive production of a single heavy meson $H$ in $e^{+} e^{-}$collisions,

$$
e^{+}+e^{-} \rightarrow V(Q) \rightarrow H(p)+X
$$

in the kinematical region where the centre-of-mass energy $Q$ of the collision is much larger than the heavy-quark (heavy-meson) mass $m$. This process is completely analogous to the process in Eq. (8), apart from the replacement of the observed light-flavoured hadron $h(p)$ by the heavy-flavoured hadron $H(p)$.

We study the inclusive cross section $d \sigma / d x$ with respect to the energy fraction $x=$ $2 p \cdot Q / Q^{2}$ of the heavy-flavoured hadron, and we define the inclusive distribution

$$
\mathcal{D}\left(x ; Q^{2}, m^{2}\right) \equiv \frac{1}{\sigma_{\text {tot }}} \frac{d \sigma}{d x},
$$

where $\sigma_{\text {tot }}$ is the total hadronic cross section in $e^{+} e^{-}$annihilation. Since we are mainly interested in the large- $x$ behaviour, we consider only the flavour non-singlet contribution to the cross section. Using the perturbative fragmentation function formalism of Eqs. (38) and (39), we write the $N$ moments of the inclusive distribution as

$$
\mathcal{D}_{N}\left(Q^{2}, m^{2}\right)=\frac{\sigma^{(L O)}}{\sigma_{\text {tot }}} C_{N}^{\left(e^{+} e^{-}\right)}\left(\alpha_{\mathrm{S}}\left(\mu^{2}\right) ; Q^{2}, \mu^{2}, \mu_{F}^{2}\right) E_{N}\left(\mu_{F}^{2}, \mu_{0 F}^{2}\right) D_{N}^{\text {ini }}\left(\alpha_{\mathrm{S}}\left(\mu_{0}^{2}\right) ; \mu_{0}^{2}, \mu_{0 F}^{2}, m^{2}\right)
$$

where the ratio $\sigma^{(L O)} / \sigma_{\text {tot }}$ is given in Eq. (37), $C_{N}^{\left(e^{+} e^{-}\right)}$is the $\overline{\mathrm{MS}}$ coefficient function in Eq. (9), $E_{N}$ is the AP evolution operator in Eq. (43), and $D_{N}^{\text {ini }}$ is the perturbative initial condition for the heavy-quark fragmentation function. To be precise, the right-hand side of Eq. (79) should contain an additional factor $D_{\mathcal{Q} / H, N}^{\mathrm{np}}$, describing the non-perturbative fragmentation of the heavy quark $\mathcal{Q}$ into the observed heavy meson $H$. Since we are mainly interested in the perturbative contributions to the inclusive distribution, we do not include this factor. 

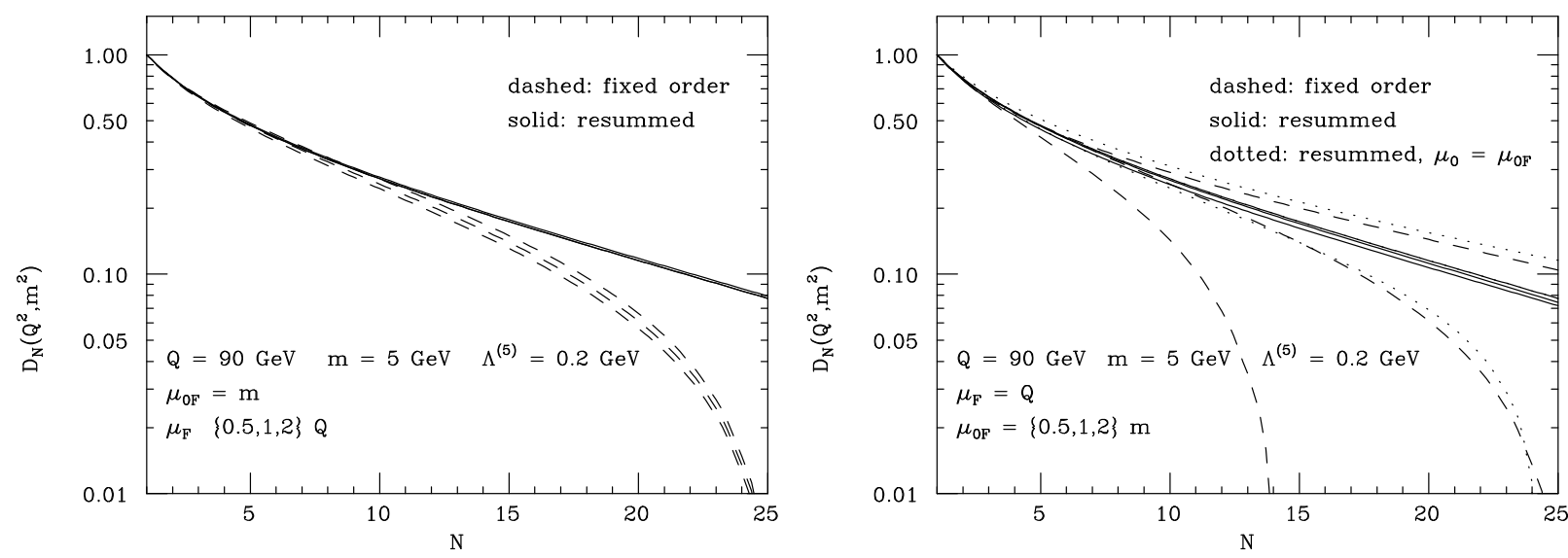

Figure 7: Dependence of the moments $\mathcal{D}_{N}$ on the variations of the factorization scales $\mu_{F}$ and $\mu_{0 F}$. The renormalization scales $\mu$ and $\mu_{0}$ are kept fixed at $Q$ and $m$ respectively, except for the dotted lines in the right-hand plot, where $\mu_{0}=\mu_{0 F}$.

In the following we compare fixed-order and resummed calculations for the inclusive distribution in Eq. (78). Analogously to the comparisons in Sects. 2.2 and 3.3, the fixedorder calculation uses the NLO expressions for $C_{N}^{\left(e^{+} e^{-}\right)}$[18, 19] and $D_{N}^{\text {ini }}$ [15], while the resummed calculation is obtained by using the expressions in Eqs. (36) and (76), which include the full NLO result and resum soft-gluon effects beyond $\mathcal{O}\left(\alpha_{\mathrm{S}}\right)$ to NLL accuracy.

We recall that soft-gluon resummation for the $e^{+} e^{-}$inclusive distribution $\mathcal{D}$ was first considered in Ref. [17]. Comparing our resummed expression with the NLL results of Ref. [17] in the ultrarelativistic limit $Q \gg m$, we find complete agreement. This should be regarded as a consistency check of our results. However, the authors of Ref. [17] did not use the fragmentation function formalism. Therefore, resummed formulae for the (processdependent) massless coefficient function $C_{N}^{\left(e^{+} e^{-}\right)}$and the (process-independent) heavy-quark initial condition $D_{N}^{\text {ini }}$ cannot be extracted separately from Ref. [17].

Since the most recent and accurate data on heavy-quark production in $e^{+} e^{-}$annihilation come from $b$-quark fragmentation at LEP and SLC, in our numerical study we choose the centre-of-mass energy $Q=90 \mathrm{GeV}$, and the heavy-quark mass $m=5 \mathrm{GeV}$.

We first consider the fixed-order and resummed calculations for the $N$ moments of the heavy-quark distribution $\mathcal{D}\left(x ; Q^{2}, m^{2}\right)$. Figure 7 shows the effect of varying the factorization scales $\mu_{F}$ and $\mu_{0 F}$ in the ranges $\{Q / 2,2 Q\}$ and $\{m / 2,2 m\}$, respectively. The left panel shows the effect of varying $\mu_{F}$, the right one that of varying $\mu_{0 F}$. We use the customary practice of performing scale variations to estimate (a lower bound on) the theoretical uncertainty due to uncalculated perturbative terms of higher order. From the plot on the left-hand side, we observe that the 'theoretical uncertainty' on the large- $N$ moments due to $\mu_{F}$ variations is smaller when resummation is performed. This effect derives from the resummation in the $e^{+} e^{-}$coefficient function $C_{N}^{\left(e^{+} e^{-}\right)}$of Eq. (79), and thus it is very similar to the effect already noticed in Sect. 2.2 for the fragmentation spectrum of light hadrons (see, e.g., Fig. 2). However, unlike those in Fig. 2, the NLO and NLO+NLL results in Fig. 7 are very different, well beyond the band due to $\mu_{F}$ variations. The difference is due 


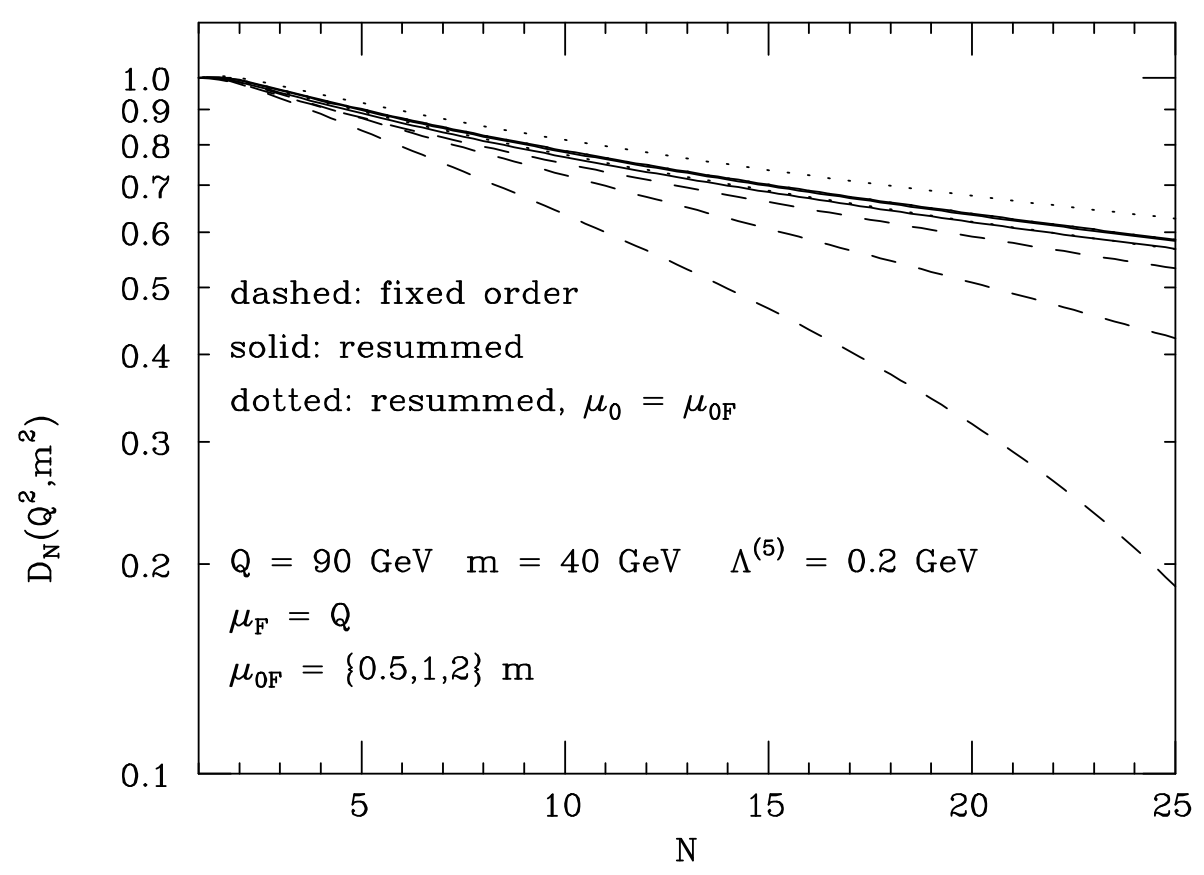

Figure 8: Dependence of the moments $\mathcal{D}_{N}$ on the variation of the factorization and renormalization scales $\mu_{0 F}$ and $\mu_{0}$ for a fictitious heavy quark of mass $m=40 \mathrm{GeV}$.

to the large effect produced by the resummation of the initial condition $D_{N}^{\text {ini }}$. Variations of the factorization scale $\mu_{0 F}$ for the initial condition are considered in the plot on the right-hand side, where we can see a very remarkable reduction in the scale dependence of the resummed calculation. Since $\mu_{0 F}$ is an auxiliary scale, introduced to perform the resummation of the large collinear terms $\alpha_{\mathrm{S}} \ln Q^{2} / \mathrm{m}^{2}$, the reduced scale dependence of the $\mathrm{NLO}+\mathrm{NLL}$ calculation implies that Sudakov resummation has highly improved collinear resummation in the large- $N$ region. If we also vary the renormalization scale $\mu_{0}$ (dotted lines in the right-hand plot), we observe an increased 'theoretical uncertainty' of the resummed calculation. This is mainly because, in the calculation of the initial condition, we are using perturbation theory down to a fairly small scale $\mu_{0}$, namely $\mu_{0} \sim m \sim 5 \mathrm{GeV}$. To show how a limiting factor to the theoretical accuracy of the calculation is the low scale set by the bottom mass, we consider a plot analogous to the right one in Fig. 7, but for a fictitious heavy quark of mass $m=40 \mathrm{GeV}$. All other parameters remain identical. In Fig. 8, we can clearly see how the 'theoretical uncertainty' is now greatly reduced by resummation, even when varying also the renormalization scale $\mu_{0}$.

To consider also the $x$-space distribution, we perform a numerical inverse Mellin transformation of Eq. (79) by using the Minimal Prescription of Ref. [28], as already described in Sects. 2.2 and 3.3. Figure 9 shows again the effect of varying the factorization scales, this time on the $x$-space inclusive distribution $\mathcal{D}\left(x ; Q^{2}, m^{2}\right)$. A smearing function $D^{\text {smear }}(x) \propto x^{0.5}(1-x)^{20}$, properly normalized so that $\int_{0}^{1} d x D^{\text {smear }}(x)=1$, has been convoluted with the purely perturbative results for better visibility. As in the case of the $N$ moments (Fig. 7), inclusion of Sudakov resummation greatly reduces the dependence on factorization-scale variations: the shape of the perturbative contribution to the inclusive distribution can now be more reliably predicted. The improvement also looks robust with 

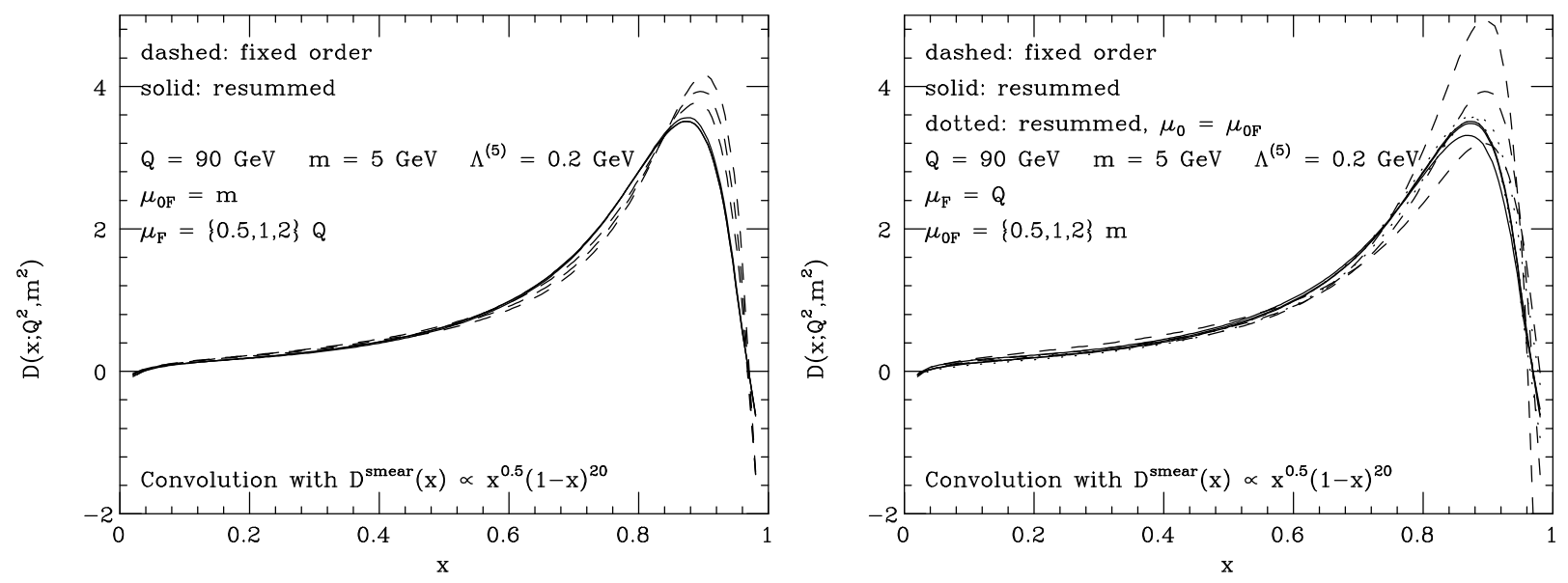

Figure 9: Dependence of the heavy-quark distribution $\mathcal{D}(x)$ on the variations of the factorization scales $\mu_{F}$ (left) and $\mu_{0 F}$ (right). Convolution with a smearing function $D^{\text {smear }}(x) \propto x^{0.5}(1-x)^{20}$, normalized to have $D_{N=1}^{\text {smear }}=1$, has been included for better visibility. The dotted lines in the right-hand plot are obtained by varying also the renormalization scale $\mu_{0}\left(\mu_{0}=\mu_{0 F}=\{0.5,2\}\right)$.

respect to variations of the renormalization scale $\mu_{0}$ (dotted lines in the right-hand plot). We can also observe that the unphysical behaviour (its origin has been discussed at the end of Sect. 3.3) very close to $x=1$, where the heavy-quark distribution turns negative, is mitigated by the inclusion of the resummed coefficient function and initial condition.

As shown by the numerical results presented in this section (and in Sect. 3.3), Sudakov resummation has important effects on heavy-quark fragmentation. In particular, the reduced scale-dependence of the resummed calculation permits a better control of the non-perturbative contributions to the heavy-quark fragmentation function. Most of these effects follow from the resummation in the perturbative initial condition and are, therefore, process-independent. For practical phenomenological purposes, one may thus think of evaluating the initial condition by considering only fixed-order perturbative contributions, without explicitly resumming Sudakov-enhanced contributions. The latter would then 'effectively' be reabsorbed in the definition of the non-perturbative component of the heavy-quark fragmentation function. However, besides it being poorly justified on theoretical grounds, such an approach would also lead to much larger uncertainties due to the much stronger scale dependence of the fixed-order calculation.

\section{Summary}

In this paper we have performed soft-gluon resummation with NLL accuracy for fragmentation processes of light and heavy quarks (hadrons) at high momentum fraction.

In the light-quark case, the fragmentation cross sections (see Eq. (1)) are obtained by convoluting process-dependent coefficient functions with the non-perturbative and processindependent fragmentation functions. We have provided the explicit resummed expressions for the non-singlet $\overline{\mathrm{MS}}$ coefficient functions of the one-particle and two-particle inclusive 
distributions in $e^{+} e^{-}$collisions and the single-particle inclusive cross section in DIS. We have studied in detail the $e^{+} e^{-}$one-particle distribution by matching (see Eq. (36)) the NLL resummed part with the complete calculation at NLO. From the mumerical comparison of our result with the NLO approximation, we observe that resummation stabilizes the calculation with respect to renormalization/factorization scales variations, and increases slightly the coefficient function in the large- $x$ region. These findings can be useful for improved phenomenological analyses and determinations of the quark fragmentation functions at large $x$.

In the heavy-quark case, the cross sections can be computed by convoluting coefficient functions, evaluated in the massless-quark approximation, with the (process-independent) perturbative fragmentation function of the heavy quark (see Eq. (38)). We have shown how the initial condition $D^{\text {ini }}(x)$, at the scale $m$, for the evolution of the perturbative fragmentation function can be obtained from the universal factorization properties of parton radiation in the collinear and quasi-collinear limits. We have then exploited this method to perform all-order resummation of the large soft-gluon contributions that dominate the behaviour of $D^{\text {ini }}(x)$ at high $x$. We have derived an expression (see Eq. (76)) for the initial condition $D^{\text {ini }}(x)$ that explicitly resums Sudakov terms up to NLL accuracy and is consistently matched with the complete NLO calculation. This result extends the LL process-independent calculation of Ref. [15] to NLL order and the NLL process-dependent calculation of Ref. [17] in a process-independent way.

Our numerical studies of heavy-quark fragmentation show that Sudakov resummation softens the high- $x$ behaviour of heavy-quark distributions. It also leads to a marked reduction of the dependence on the renormalization/factorization scales, making the perturbative predictions for heavy-quark fragmentation processes more reliable. These perturbative features are important for improved studies of the non-perturbative component that has to be included in phenomenological applications to heavy-quark fragmentation.

\section{Appendices}

\section{A Two-particle inclusive distribution in $e^{+} e^{-}$annihilation}

In this appendix we study the less inclusive case of $e^{+} e^{-}$annihilation with two observed hadrons, $h_{A}$ and $h_{B}$, in the final state (Fig. 10):

$$
e^{+}+e^{-} \rightarrow V(Q) \rightarrow h_{A}\left(p_{1}\right)+h_{B}\left(p_{2}\right)+X
$$

We consider the corresponding two-particle inclusive distribution,

$$
\mathcal{D}\left(x_{1}, x_{2} ; Q^{2}\right) \equiv \frac{1}{\sigma_{\text {tot }}} \frac{d \sigma_{h_{A} h_{B}}^{\left(e^{+} e^{-}\right)}}{d x_{1} d x_{2}},
$$




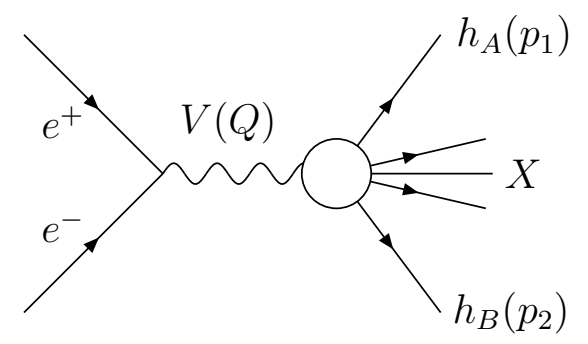

Figure 10: Inclusive production of two hadrons with momenta $p_{1}$ and $p_{2}$ in $e^{+} e^{-}$annihilation.

where $\sigma_{\text {tot }}$ is the total hadronic cross section, and the kinematic variables $x_{1}$ and $x_{2}$ are defined according to Ref. [18]:

$$
x_{1} \equiv \frac{2 p_{1} \cdot Q}{Q^{2}}, \quad x_{2} \equiv \frac{p_{1} \cdot p_{2}}{p_{1} \cdot Q} .
$$

Note that $x_{1}$ and $x_{2}$ can independently vary in the whole kinematical range between 0 and 1. In the $e^{+} e^{-}$centre-of-mass frame, $x_{1}$ coincides with the energy fraction of the hadron with momentum $p_{1}$, while $x_{2}$ is the energy fraction of the hadron with momentum $p_{2}$ times $1-\cos \theta_{12}, \theta_{12}$ being the angle between the two hadrons. The choice of these two kinematic variables guarantees that the two-particle distribution is collinear safe with respect to final-state QCD radiation for any values $x_{1}, x_{2} \neq 0$. Considering sufficiently large values of both $x_{1}$ and $x_{2}$, we ensure that $\theta_{12}$ is large and, hence, the two observed hadrons belong to two different jets in a nearly back-to-back configuration.

More precisely, owing to our definition of the kinematic variables $x_{1}$ and $x_{2}$, the twoparticle distribution $\mathcal{D}\left(x_{1}, x_{2} ; Q^{2}\right)$ can be computed according to the following QCD factorization formulain

$$
\begin{aligned}
\mathcal{D}\left(x_{1}, x_{2} ; Q^{2}\right)= & \frac{\sigma^{(L O)}}{\sigma_{\text {tot }}} \int_{x_{1}}^{1} \frac{d z_{1}}{z_{1}} \int_{x_{2}}^{1} \frac{d z_{2}}{z_{2}} C^{\left(e^{+} e^{-}\right)}\left(x_{1} / z_{1}, x_{2} / z_{2}, \alpha_{\mathrm{S}}\left(\mu^{2}\right) ; Q^{2}, \mu^{2}, \mu_{F}^{2}\right) \\
& \cdot D\left(z_{1}, \mu_{F}^{2}\right) D\left(z_{2}, \mu_{F}^{2}\right),
\end{aligned}
$$

where $D\left(x, \mu_{F}^{2}\right)$ is the same fragmentation function as appears in Eq. (9). The two-particle coefficient function $C^{\left(e^{+} e^{-}\right)}\left(x_{1}, x_{2}\right)$ is computable in QCD perturbation theory. In the naïve parton model (i.e. at the $\mathrm{LO}$ ), we have $C^{\left(e^{+} e^{-}\right)}\left(x_{1}, x_{2}\right)=\delta\left(1-x_{1}\right) \delta\left(1-x_{2}\right)$ and, thus, the two-particle distribution is simply given by the product $D\left(x_{1}, Q^{2}\right) D\left(x_{2}, Q^{2}\right) \propto$ $\left(d \sigma / d x_{1}\right)\left(d \sigma / d x_{2}\right)$ of the two corresponding single-particle distributions in Eq. (9). Higherorder perturbative contributions lead to QCD correlation effects that spoil this naïve factorized structure and whose size is measured by the two-particle coefficient function.

\footnotetext{
$\S_{\text {Since }} x_{1}$ and $x_{2}$ are not symmetric variables with respect to $p_{1} \leftrightarrow p_{2}$, a corresponding symmetrization, $h_{A} \leftrightarrow h_{B}$, is understood in the definition of the two-particle distribution in Eq. (81).

"If one uses the energy fractions $2 p_{1} Q / Q^{2}, 2 p_{2} Q / Q^{2}$ of the two hadrons in the definition of the distribution, an additional physical cutoff has to be introduced to avoid the kinematic region where $p_{1}$ and $p_{2}$ are collinear.

$\|$ The ratio $\sigma^{(L O)} / \sigma_{\text {tot }}$ is introduced on the right-hand side to make the notation in Eq. (83) consistent with that in Eq. (9).
} 
The complete evaluation of $C^{\left(e^{+} e^{-}\right)}\left(x_{1}, x_{2}\right)$ at the NLO was performed in [18. As in the case of the single-particle coefficient function in Eq. (11), the flavour non-singlet contributions to $C^{\left(e^{+} e^{-}\right)}\left(x_{1}, x_{2}\right)$ contain logarithmically-enhanced terms in the semi-inclusive limit $x_{1}, x_{2} \rightarrow 1$. These terms are conveniently singled out by introducing double Mellin moments as

$$
\mathcal{D}_{N_{1} N_{2}}\left(Q^{2}\right) \equiv \int_{0}^{1} d x_{1} x_{1}^{N_{1}-1} \int_{0}^{1} d x_{2} x_{2}^{N_{2}-1} \mathcal{D}\left(x_{1}, x_{2} ; Q^{2}\right)
$$

and studying their large- $N_{i}(i=1,2)$ behaviour. In $N$-moment space Eq. (83) becomes

$$
\mathcal{D}_{N_{1} N_{2}}\left(Q^{2}\right)=\frac{\sigma^{(L O)}}{\sigma_{\text {tot }}} C_{N_{1} N_{2}}^{\left(e^{+} e^{-}\right)}\left(\alpha_{\mathrm{S}}\left(\mu^{2}\right) ; Q^{2}, \mu^{2}, \mu_{F}^{2}\right) D_{N_{1}}\left(\mu_{F}^{2}\right) D_{N_{2}}\left(\mu_{F}^{2}\right)
$$

and in the limit $N_{i} \rightarrow \infty(i=1,2)$ the coefficient function $C_{N_{1} N_{2}}^{\left(e^{+} e^{-}\right.}$has a perturbative expansion similar to Eq. (7), with $\alpha_{\mathrm{S}}^{n} \ln ^{m} N$ replaced by $\alpha_{\mathrm{S}}^{n} \ln ^{m_{1}} N_{1} \ln ^{m_{2}} N_{2}\left(m_{1}+m_{2} \leq 2 n\right)$.

All-order resummation of the logarithmically-enhanced terms can be performed by using standard techniques. In particular, we can exploit the strict kinematical analogy with the differential Drell-Yan (DY) distribution, and we can proceed as in Sect. 4 of the first paper in Ref. [9]. We obtain the exponentiated result

$$
\begin{aligned}
& C_{N_{1} N_{2}}^{\left(e^{+} e^{-}\right)}\left(\alpha_{\mathrm{S}}\left(\mu^{2}\right) ; Q^{2}, \mu^{2}, \mu_{F}^{2}\right)=\exp \left\{\left[\int_{0}^{1} d x \frac{x^{N_{1}-1}-1}{1-x} \int_{\mu_{F}^{2}}^{(1-x) Q^{2}} \frac{d q^{2}}{q^{2}} A\left[\alpha_{\mathrm{S}}\left(q^{2}\right)\right]+\left(N_{1} \leftrightarrow N_{2}\right)\right]\right. \\
& \quad+\int_{0}^{1} d x_{1} \int_{0}^{1} d x_{2} \frac{\left(x_{1}^{N_{1}-1}-1\right)}{1-x_{1}} \frac{\left(x_{2}^{N_{2}-1}-1\right)}{1-x_{2}} A\left[\alpha_{\mathrm{S}}\left(\left(1-x_{1}\right)\left(1-x_{2}\right) Q^{2}\right)\right] \\
& \left.\quad+\mathcal{O}\left(\alpha_{\mathrm{S}}\left(\alpha_{\mathrm{S}} \ln N_{i}\right)^{n}\right)\right\} .
\end{aligned}
$$

The structure of Eq. (86) is similar to that of Eqs. (14)-(16) for the single-particle coefficient function. The main difference is that the integrands of Eq. (86) contain a single perturbative function, namely the soft-gluon function $A\left(\alpha_{\mathrm{S}}\right)$ given by Eq. (17). This is due to the fact that, when $x_{1}$ and $x_{2}$ are both large, only the emission of soft gluons is kinematically allowed in the final state.

The perturbative expansion of Eq. (86) at $\mathcal{O}\left(\alpha_{\mathrm{S}}\right)$ agrees with the NLO result of Ref. [18]. Owing to the knowledge of the first two coefficients (see Eq. (19)) of the function $A\left(\alpha_{\mathrm{S}}\right)$, the result in Eq. (86) resums the LL $\left(m_{1}+m_{2}=n+1\right)$ and NLL $\left(m_{1}+m_{2}=n\right)$ terms $\alpha_{\mathrm{S}}^{n} \ln ^{m_{1}} N_{1} \ln ^{m_{2}} N_{2}$ in the exponent of the two-particle coefficient function $C_{N_{1} N_{2}}^{\left(e^{+} e^{-}\right)}$. The integrals in Eq. (86) can explicitly be performed up to NLL accuracy, and the resummed coefficient function can be written in the following equivalent form:

$$
\begin{aligned}
C_{N_{1} N_{2}}^{\left(e^{+} e^{-}\right)}\left(\alpha_{\mathrm{S}}\left(\mu^{2}\right) ; Q^{2}, \mu^{2}, \mu_{F}^{2}\right) & =\exp \left[\left(\ln N_{1}+\ln N_{2}\right) g_{2 p}^{(1)}\left(\lambda_{12}\right)\right. \\
& \left.+g_{2 p}^{(2)}\left(\lambda_{12}, Q^{2} / \mu^{2} ; Q^{2} / \mu_{F}^{2}\right)+\mathcal{O}\left(\alpha_{\mathrm{S}}\left(\alpha_{\mathrm{S}} \ln N_{i}\right)^{n}\right)\right]
\end{aligned}
$$

where

$$
\lambda_{12}=b_{0} \alpha_{\mathrm{S}}\left(\mu^{2}\right)\left(\ln N_{1}+\ln N_{2}\right)
$$




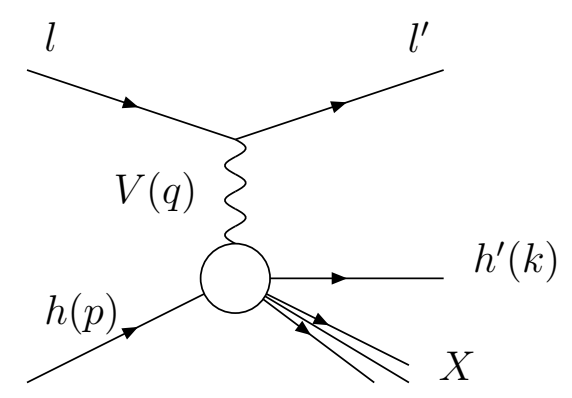

Figure 11: Inclusive production of a single hadron $h^{\prime}$ with momentum $k$ in deep inelastic leptonhadron scattering.

and the LL and NLL functions $g_{2 p}^{(1)}$ and $g_{2 p}^{(2)}$ are given by

$$
\begin{aligned}
g_{2 p}^{(1)}\left(\lambda_{12}\right) & =\frac{A^{(1)}}{\pi b_{0} \lambda_{12}}\left[\lambda_{12}+\left(1-\lambda_{12}\right) \ln \left(1-\lambda_{12}\right)\right] \\
g_{2 p}^{(2)}\left(\lambda_{12}, Q^{2} / \mu^{2} ; Q^{2} / \mu_{F}^{2}\right) & =\frac{A^{(1)} b_{1}}{2 \pi b_{0}^{3}}\left[2 \lambda_{12}+2 \ln \left(1-\lambda_{12}\right)+\ln ^{2}\left(1-\lambda_{12}\right)\right] \\
& -\frac{1}{\pi b_{0}}\left[\lambda_{12}+\ln \left(1-\lambda_{12}\right)\right]\left(\frac{A^{(2)}}{\pi b_{0}}-A^{(1)} \ln \frac{Q^{2}}{\mu^{2}}\right) \\
& -\frac{2 A^{(1)} \gamma_{E}}{\pi b_{0}} \ln \left(1-\lambda_{12}\right)-\frac{A^{(1)}}{\pi b_{0}} \lambda_{12} \ln \frac{Q^{2}}{\mu_{F}^{2}} .
\end{aligned}
$$

The coefficient functions on the right-hand side of the factorization formulae in Eqs. (9) and (83) are factorization-scheme-dependent. The resummed expressions in Eqs. (23) and (86) are valid in the $\overline{\mathrm{MS}}$ factorization scheme. The ratio

$$
\Delta_{N_{1} N_{2}}^{\left(e^{+} e^{-}\right)}\left(\alpha_{\mathrm{S}}\left(\mu^{2}\right) ; Q^{2}, \mu^{2}\right) \equiv \frac{C_{N_{1} N_{2}}^{\left(e^{+} e^{-}\right)}\left(\alpha_{\mathrm{S}}\left(\mu^{2}\right) ; Q^{2}, \mu^{2}, \mu_{F}^{2}\right)}{C_{N_{1}}^{\left(e^{+} e^{-}\right)}\left(\alpha_{\mathrm{S}}\left(\mu^{2}\right) ; Q^{2}, \mu^{2}, \mu_{F}^{2}\right) C_{N_{2}}^{\left(e^{+} e^{-}\right)}\left(\alpha_{\mathrm{S}}\left(\mu^{2}\right) ; Q^{2}, \mu^{2}, \mu_{F}^{2}\right)}
$$

between two-particle and one-particle coefficient functions is instead independent of the factorization scheme. This ratio corresponds to the two-particle coefficient function as defined in the alternative factorization scheme**, named 'annihilation scheme' [19], introduced in Ref. 18]. Using our NLL resummed expressions for $C_{N_{1} N_{2}}^{\left(e^{+} e^{-}\right)}$and $C_{N}^{\left(e^{+} e^{-}\right)}$, it is straightforward to check that the dependence on the factorization scale $\mu_{F}$ consistently cancels in the ratio $\Delta_{N_{1} N_{2}}^{\left(e^{+} e^{-}\right)}$, i.e. in the right-hand side of Eq. (91).

\section{B Single-particle inclusive distribution in lepton-hadron collisions}

We conclude our analysis of light-hadron fragmentation in the semi-inclusive region by considering deep inelastic lepton-hadron scattering (DIS). Instead of studying the DIS total

** The annihilation scheme amounts to redefining the $\overline{\mathrm{MS}}$ fragmentation function in such a way that the corresponding coefficient function in Eq. (9) is equal to unity. 
cross section, we are interested in the inclusive production of a single final-state hadron (Fig. 11):

$$
l+h(p) \rightarrow l^{\prime}+h^{\prime}(k)+X
$$

To study the fragmentation of the tagged hadron at high momentum fraction, we can limit ourselves to considering the approximation in which the scattering process in Eq. (92) occurs through the exchange of a single vector boson with momentum $q$ and hardness $-q^{2}=Q^{2}>0$. We denote by $p$ and $k$ the momenta of the incoming and final-state hadrons, respectively.

Besides the customary Bjorken variable $x_{B}$, we define the final-state variable $z$ as follows 18]:

$$
x_{B} \equiv \frac{Q^{2}}{2 p \cdot q}, \quad z \equiv \frac{p \cdot k}{p \cdot q},
$$

and the semi-inclusive region we are interested in is specified by the limit $x_{B} \rightarrow 1$ and $z \rightarrow 1$.

In the Breit frame, where $q^{\mu}=(0, \mathbf{0},-Q)$ and $p^{\mu}=(1, \mathbf{0}, 1) Q /\left(2 x_{B}\right)$, we have $z=$ $(1-\cos \theta) k_{0} / Q$, so the variable $z$ is related to the energy $k_{0}$ of the fragmenting hadron and to its scattering angle $\theta$ with respect to $p$. When approaching the semi-inclusive limit, the two hadrons $h(p)$ and $h^{\prime}(k)$ are in a back-to-back configuration, with the target hadron momentum $p^{\mu} \simeq(1, \mathbf{0}, 1) Q / 2$ going forward and the fragmenting hadron momentum $k^{\mu} \simeq(1, \mathbf{0},-1) Q / 2$ moving backward in the current-jet hemisphere.

Note that $x_{B}$ and $z$ can independently vary in the whole kinematical range between 0 and 1. Moreover, as long as $z$ is not vanishing, the fragmenting hadron $h^{\prime}(k)$ cannot become collinear to the colliding hadron $h(p)$. Therefore, the single-inclusive cross section $d \sigma_{h^{\prime}}^{(\mathrm{DIS})} / d x_{B} d z$ fulfils [18] the following QCD factorization formula:

$$
\begin{aligned}
\frac{d \sigma_{h^{\prime}}^{(\mathrm{DIS})}}{d x_{B} d z}= & \sigma^{(0)} \int_{x_{B}}^{1} \frac{d x}{x} \int_{z}^{1} \frac{d y}{y} C^{(\mathrm{DIS})}\left(x, y, \alpha_{\mathrm{S}}\left(\mu^{2}\right) ; Q^{2}, \mu^{2}, \mu_{F}^{2}\right) \\
\cdot & F\left(x_{B} / x, \mu_{F}^{2}\right) D\left(z / y, \mu_{F}^{2}\right),
\end{aligned}
$$

where $\sigma^{(0)}$ is the LO cross section, $D\left(z, \mu_{F}^{2}\right)$ is the parton fragmentation function of the tagged hadron $h^{\prime}(k)$ and $F\left(x, \mu_{F}^{2}\right)$ is the parton distribution function of the colliding hadron $h(p)$. To be defined, we recall [4] that $F\left(x, \mu_{F}^{2}\right)$ appears in the analogous factorization formula for the DIS total cross section $d \sigma^{(\mathrm{DIS})} / d x_{B}$ :

$$
\frac{d \sigma^{(\mathrm{DIS})}}{d x_{B}}=\sigma^{(0)} \int_{x_{B}}^{1} \frac{d x}{x} C^{(\mathrm{DIS})}\left(x, \alpha_{\mathrm{S}}\left(\mu^{2}\right) ; Q^{2}, \mu^{2}, \mu_{F}^{2}\right) F\left(x_{B} / x, \mu_{F}^{2}\right) .
$$

Since eventually we are mainly interested in the semi-inclusive limit, we omit parton indices in Eqs. (94) and (95), and we understand that the parton fragmentation function and distribution function refer to the flavour non-singlet components.

The single-inclusive coefficient function $C^{(\mathrm{DIS})}(x, y)$ is computable in QCD perturbation theory. In the naïve parton model (i.e. at the LO), we have $C^{(\mathrm{DIS})}(x, y)=\delta(1-x) \delta(1-y)$, 
and the single-inclusive cross section in Eq. (94) is simply proportional to the product of the parton distribution and the fragmentation function, $d \sigma_{h^{\prime}}^{(\text {(IS) }} / d x_{B} d z \propto F\left(x_{B}, Q^{2}\right) D\left(z, Q^{2}\right)$. The complete NLO calculation of $C^{(\mathrm{DIS})}(x, z)$ was performed in Ref. [18].

As in the case of the $e^{+} e^{-}$two-particle coefficient function $C^{\left(e^{+} e^{-}\right)}\left(x_{1}, x_{2}\right)$ in Eq. (83), at high perturbative orders the flavour non-singlet contribution to $C^{(\mathrm{DIS})}\left(x_{B}, z\right)$ contains terms that are logarithmically enhanced in the semi-inclusive limit $x_{B}, z \rightarrow 1$. Similarly to the two-particle distribution in $e^{+} e^{-}$annihilation, it is thus convenient to define the double Mellin moments:

$$
C_{N_{1} N_{2}}^{(\mathrm{DIS})}\left(\alpha_{\mathrm{S}}\left(\mu^{2}\right) ; Q^{2}, \mu^{2}, \mu_{F}^{2}\right) \equiv \int_{0}^{1} d x x^{N_{1}-1} \int_{0}^{1} d z z^{N_{2}-1} C^{(\mathrm{DIS})}\left(x, z, \alpha_{\mathrm{S}}\left(\mu^{2}\right) ; Q^{2}, \mu^{2}, \mu_{F}^{2}\right),
$$

and to consider their large- $N_{i}(i=1,2)$ behaviour.

The general structure of $C_{N_{1} N_{2}}^{(\text {DIS }}$ in the large- $N_{i}$ limit is similar to that of $C_{N_{1} N_{2}}^{\left(e^{+} e^{-}\right)}$and the resummation of the large logarithmic contributions can be performed following Ref. [9]. Using the $\overline{\mathrm{MS}}$ factorization scheme, to NLL order we find

$$
\ln C_{N_{1} N_{2}}^{(\mathrm{DIS})}\left(\alpha_{\mathrm{S}}\left(\mu^{2}\right) ; Q^{2}, \mu^{2}, \mu_{F}^{2}\right)=\ln C_{N_{1} N_{2}}^{\left(e^{+} e^{-}\right)}\left(\alpha_{\mathrm{S}}\left(\mu^{2}\right) ; Q^{2}, \mu^{2}, \mu_{F}^{2}\right)+\mathcal{O}\left(\alpha_{\mathrm{S}}\left(\alpha_{\mathrm{S}} \ln N_{i}\right)^{n}\right),
$$

with $C_{N_{1} N_{2}}^{\left(e^{+} e^{-}\right)}$given by the expression in Eq. (86). This result has a simple physical explanation. In the semi-inclusive limit, the kinematical configuration of the DIS single-inclusive cross section is related to that of the $e^{+} e^{-}$two-particle cross section by crossing the DIS incoming hadron to the final state. In both cases the cross section is dominated by purely soft emission. The relation (97) thus follows from the fact that the intensities of soft-gluon radiation from space-like and time-like partons are equal up to NLL accuracy (see Ref. [7] and the discussion at the end of Sect. 2.1).

Acknowledgments. We wish to thank Luca Trentadue for participation in early stages of this work. M.C. thanks Ugo Aglietti for many conversations on this subject.

\section{References}

[1] S. Catani et al., hep-ph/0005025, in the Proceedings of the CERN Workshop on Standard Model Physics (and more) at the LHC, Eds. G. Altarelli and M.L. Mangano (CERN 2000-04, Geneva, 2000), p. 1.

[2] B. R. Webber, in Proc. of the 19th Int. Symp. on Photon and Lepton Interactions at High Energy LP99, Eds. J.A. Jaros and M.E. Peskin, Int. J. Mod. Phys. A15S1 (2000) 577 .

[3] See the fragmentation web pages of the European network "Hadronic Physics with High Energy Electromagnetic Probes" (HaPHEEP): http://droide1.pv.infn.it/FFdatabase/fragmentation.htm,

[4] R.K. Ellis, W.J. Stirling and B.R. Webber, QCD and collider physics (Cambridge University Press, Cambridge, 1996) and references therein. 
[5] G. Curci, W. Furmanski and R. Petronzio, Nucl. Phys. B175 (1980) 27; W. Furmanski and R. Petronzio, Z. Phys. C11 (1982) 293; J. Kalinowski, K. Konishi, P.N. Scharbach and T.R. Taylor, Nucl. Phys. B181 (1981) 253; E.G. Floratos, C. Kounnas and R. Lacaze, Phys. Lett. 98B (1981) 89; I. Antoniadis and E.G. Floratos, Nucl. Phys. B191 (1981) 217.

[6] J. Kodaira and L. Trentadue, Phys. Lett. 112B (1982) 66; C.T.H. Davies and J. Stirling, Nucl. Phys. B244 (1984) 337; S. Catani, E. d'Emilio and L. Trentadue, Phys. Lett. 211B (1988) 335.

[7] S. Catani, G. Marchesini and B.R. Webber, Nucl. Phys. B349 (1991) 635.

[8] G. Sterman, Nucl. Phys. B281 (1987) 310.

[9] S. Catani and L. Trentadue, Nucl. Phys. B327 (1989) 323, Nucl. Phys. B353 (1991) 183.

[10] G.P. Korchemsky, Mod. Phys. Lett. A4 (1989) 1257.

[11] N. Kidonakis and G. Sterman, Nucl. Phys. B505 (1997) 321.

[12] R. Bonciani, S. Catani, M. L. Mangano and P. Nason, Nucl. Phys. B529 (1998) 424.

[13] E. Laenen, G. Oderda and G. Sterman, Phys. Lett. B438 (1998) 173.

[14] S. Catani, M. L. Mangano and P. Nason, JHEP 9807 (1998) 024.

[15] B. Mele and P. Nason, Nucl. Phys. B361 (1991) 626.

[16] M. Cacciari and M. Greco, Nucl. Phys. B421 (1994) 530.

[17] Y. L. Dokshitzer, V. A. Khoze and S. I. Troian, Phys. Rev. D53 (1996) 89.

[18] G. Altarelli, R.K. Ellis, G. Martinelli and S.Y. Pi, Nucl. Phys. B160 (1979) 301.

[19] P. Nason and B. R. Webber, Nucl. Phys. B421 (1994) 473 (E ibid. B480 (1996) 755).

[20] P. J. Rijken and W. L. van Neerven, Phys. Lett. B392 (1997) 207, Nucl. Phys. B487 (1997) 233.

[21] Yu.L. Dokshitser, V.A. Khoze, A.H. Mueller and S.I. Troian, Basics of Perturbative QCD (Editions Frontières, Gif-sur-Yvette, 1991) and references therein.

[22] J. M. Campbell and E. W. Glover, Nucl. Phys. B527 (1998) 264; S. Catani and M. Grazzini, Phys. Lett. B446 (1999) 143, Nucl. Phys. B570 (2000) 287.

[23] Z. Bern, V. Del Duca and C. R. Schmidt, Phys. Lett. B445 (1998) 168; Z. Bern, V. Del Duca, W. B. Kilgore and C. R. Schmidt, Phys. Rev. D60 (1999) 116001; D. A. Kosower and P. Uwer, Nucl. Phys. B563 (1999) 477; S. Catani and M. Grazzini, Nucl. Phys. B591 (2000) 435

[24] D. de Florian and M. Grazzini, Phys. Rev. Lett. 85 (2000) 4678.

[25] S. Catani, D. de Florian and M. Grazzini, JHEP 0105 (2001) 025. 
[26] A. Vogt, Phys. Lett. B497 (2001) 228.

[27] V.N. Gribov and L.N. Lipatov, Sov. J. Nucl. Phys. 15 (1972) 438, Sov. J. Nucl. Phys. 15 (1972) 675.

[28] S. Catani, M. L. Mangano, P. Nason and L. Trentadue, Phys. Lett. B378 (1996) 329, Nucl. Phys. B478 (1996) 273.

[29] S. Kretzer, Phys. Rev. D62 (2000) 054001; B. A. Kniehl, G. Kramer and B. Potter, Nucl. Phys. B582 (2000) 514; L. Bourhis, M. Fontannaz, J. P. Guillet and M. Werlen, Eur. Phys. J. C19 (2001) 89.

[30] M. Cacciari, M. Greco and P. Nason, JHEP 9805 (1998) 007; F. I. Olness, R. J. Scalise and W. Tung, Phys. Rev. D59 (1999) 014506.

[31] M. Cacciari, M. Greco, B. A. Kniehl, M. Kramer, G. Kramer and M. Spira, Nucl. Phys. B466 (1996) 173; M. Cacciari, S. Frixione and P. Nason, JHEP 0103 (2001) 006.

[32] G. Colangelo and P. Nason, Phys. Lett. B285 (1992) 167.

[33] M. Cacciari and M. Greco, Phys. Rev. D55 (1997) 7134; M. Cacciari, M. Greco, S. Rolli and A. Tanzini, Phys. Rev. D55 (1997) 2736

[34] P. Nason and C. Oleari, Nucl. Phys. B565 (2000) 245.

[35] C. Peterson, D. Schlatter, I. Schmitt and P. Zerwas, Phys. Rev. D27 (1983) 105.

[36] R. L. Jaffe and L. Randall, Nucl. Phys. B412 (1994) 79.

[37] E. Braaten, K. Cheung, S. Fleming and T. C. Yuan, Phys. Rev. D51 (1995) 4819.

[38] P. Nason and B. R. Webber, Phys. Lett. B395 (1997) 355.

[39] G. P. Korchemsky and G. Sterman, Nucl. Phys. B555 (1999) 335; U. Aglietti, preprint CERN-TH/2001-050 hep-ph/0103002]; E. Gardi and J. Rathsman, preprint CERNTH/2001-083 hep-ph/0103217.

[40] D. Amati, R. Petronzio and G. Veneziano, Nucl. Phys. B140 (1978) 54, Nucl. Phys. B146 (1978) 29; R.K. Ellis, H. Georgi, M. Machacek, H.D. Politzer and G.G. Ross, Phys. Lett. 78B (1978) 281, Nucl. Phys. B152 (1979) 285.

[41] S. Catani, S. Dittmaier and Z. Trocsanyi, Phys. Lett. B500 (2001) 149.

[42] S. Keller and E. Laenen, Phys. Rev. D59 (1999) 114004. 